\title{
Market Arbitage, Social Choice and the Core
}

\author{
by \\ Graciela Chichilnisky, Columbia University
}

July 1996

Discussion Paper Series No. 9596-30

$d p 9596-30$ 


\title{
Market Arbitrage, Social Choice and the Core
}

\author{
Graciela Chichilnisky*
}

December 1991, revised December 1993 and July 1996

\begin{abstract}
This paper establishes a clear connection between equilibrium theory, game theory and social choice theory by showing that, for a well defined social choice problem, a condition which is necessary and sufficient to solve this problem-limited arbitrage - is the same as the condition which is necessary and sufficient to establish the existence of an equilibrium and the core. The connection is strenghtened by establishing that a market allocation, which is in the core, can always be realized as a social allocation, i.e. an allocation which is optimal according to an ordering chosen by a social choice rule. Limited arbitrage characterizes those economies without Condorcet triples, and those for which Arrow's paradox can be resolved on choices of large utility values.
\end{abstract}

\section{Introduction}

Markets provide a widely used solution to the problem of allocating resources among the members of the economy. A market equilibrium is individually optimal and clears the markets. The efficiency of competitive market allocations is what makes them desirable.

A different solution to the resource allocation problem is provided by social choice rules. These rules derive a social preference as a function of individual preferences in a fair fashion, for example through voting. An allocation which is optimal among all feasible allocations according to a social preference, is called a social allocation.

A third solution to the problem of resource allocations is to seek allocations in the core: these are allocations from which no coalition would want to deviate.

This paper establishes a clear connection between equilibrium theory, social choice theory and game theory by showing that, for a well defined social choice problem, a condition which is necessary and sufficient to solve this problem-limited arbitrage -is the same as the condition which is necessary and sufficient to establish the existence of an equilibrium and the core. ${ }^{1}$ The connection is strengthened by establishing that a market allocation, which is in the core, can always be realized as a social allocation.

*UNESCO Chair in Mathematics and Economics and Director, Program on Information and Resources, Columbia University. mascc2.tex. email: gc9@columbia.edu. Support from Stanford Institute for Theoretical Economics, NSF Grant SES92-16028, the Sloan Foundation, the Salinbemi Chair at the University of Siena and the Leif Johansen Award at the University of Oslo contributed to the research reported here. The main results of this paper were presented at “Columbia Celebrates Arrow's Contributions" at Columbia University, October 1991, and at invited addresses to the International Congress of Mathematicians Paris, July 4, 1994, a Colloquium at the University of California at Berkeley, March 1993, at the American Economic Association Annual Meetings in Boston, January 3-5 1994, and as a keynote address to the World Congress on Non Linear Analysis in Athens, July 16, 1996.

${ }^{1}$ These results have been announced and presented in a number of papers starting in 1992: Chichilnisky [18], [16], [17], [22], [21], [24], [20], [26], [30], [27] Chichilnisky and Heal [31]; these papers and the literature are discussed in a comment by Monteiro Page and Wooders [50] and its response Chichilnisky [25]. 
How does limited arbitrage work? Limited arbitrage is a condition on traders' endowments and preferences. Introduced in [18] it bounds potential gains from trade, and the scope for mutually advantageous reallocation in the economy. In mathematical terms, limited arbitrage is the non-empty intersection of a family of cones. Somewhat surprisingly, this nonempty intersection is the same as a topological condition: the contractibility of spaces of preferences. This connection arises from a new result: a family of convex sets intersects if and only if every subfamily has a contractible union, see [15], [19]. It has been shown that contractibility is a restriction on the diversity of preferences [43]. Through its connection with contractibility, therefore, limited arbitrage implies a restriction on the traders' diversity [22]. Indeed, the central role of limited arbitrage in resource allocation is explained by its connection with social diversity [22]. Social diversity is crucial for resource allocation.

For example, social diversity in the form of contractibility of preferences is necessary and sufficient for the existence of continuous anonymous social choice rules respecting unanimity, Chichilnisky and Heal [32],[18],,21]. Since limited arbitrage is a form of contractibility, this explains the connection between limited arbitrage and social choice. I show below that limited arbitrage is also closely connected with Arrow's impossibility theorem: it is necessary and sufficient to eliminate Condorcet triples - or cyclical behavioron choices over feasible allocations of utility value which approaches the supremum of utilities, and for solving Arrow's theorem on such choices. ${ }^{2}$

Diversity has a fundamental role in other forms of resource allocation. Indeed, markets exist because people are different. Only when traders have different preferences and endowments do they have a reason to trade. Furthermore a condition which limits diversity, namely limited arbitrage, is necessary and sufficient for the existence of a competitive equilibrium in general economies [16], [31], [24], [26]. It is also necessary and sufficient for the uniqueness of equilibrium in strictly regular economies [27]. Elsewhere I showed also that the diversity of the traders is necessary and sufficient for the existence of Pareto efficient allocations [28], [29], [16], [24]. Thus a failure of limited arbitrage prevents optimal coalition formation: it leads to an empty core [21]. Diversity is therefore equally important for game theoretical allocations.

These results highlight novel features of resource allocation. For example, market allocations are generally considered to be practical, more so than social allocations. One reason for this is that markets are viewed as having equilibria generally, while social choice theory has always stressed paradoxes and non-existence results. Our results, however, show that this is not accurate. The same restriction on diversity is necessary for the existence of market equilibrium and for the existence of appropriate social choice rules. ${ }^{3}$

A careful examination of the literature illustrates the importance of diversity in resource allocation. The social choice literature has focused on interpersonal diversity as a reason for the non-existence of social choice rules. Several ways of resolving social choice problems have been proposed by limiting the diversity of the individual preferences, a 'domain restriction' on preferences, Black [8][9], Pattanaik and Sen [54], and Chichilnisky and Heal [32]. These works propose ways of limiting the diversity of individuals. The issue of existence of universal social choice rules-a problem which in its more general

\footnotetext{
${ }^{2}$ This is an ordinal result, which is independent from the utility representation of preferences. See also [21], [20], and [22].

${ }^{3}$ Competitive equilibria are not always available: when the traders endowments and preferences are very different the economy can fail to have a competitive equilibrium. Arrow and Hahn ([3], Chapter $4,1)$ provided examples of standard market economies with no competitive equilibrium. Their example highlights the role of interpersonal differences between the traders, differences in their endow ments and in their preferences, in preventing the existence of a competitive equilibrium. The failure of existence arises when some traders have zero income, while others wish and can afford unboundedly large allocations which a bounded economy cannot accommodate ([3], Chapter 4, p. 80).
} 
form has no solution-is turned into the question: for what societies can the social choice problem be resolved? Or: how much diversity can a society function with? ${ }^{4}$

The issue of diversity has also been studied in the context of market equilibrium. There are widely used conditions which ensure the existence of a competitive equilibrium. These are also restrictions in social diversity: they require that the endowments of any household are desired, indirectly or directly, by others, so that their incomes cannot fall to zero: examples are McKenzie's irreducibility condition [47][48][49], and the resource relatedness condition in Arrow and Hahn [3]. These conditions are different from limited arbitrage, but nevertheless restrict the diversity of individuals' preferences and endowments. Limited arbitrage provides another limitation in social diversity: it does so parsimoniously, because it is a minimal condition, in the sense of being necessary as well as sufficient for existence. In addition, it applies simultaneously to resource allocation by market equilibrium, by social choice and the core. Indoed limited arbitrage measures precisely the amount of diversity acceptable for the functioning of markets, cooperative games and social choice.

There is a different perspective on diversity, one which views diversity as a positive factor in a group's adaptation to its environment. This view contrasts with the role of diversity on resource allocation, in which the functioning of economic institutions imposes limits on diversity. The contrast suggests an open, and perhaps unsettling, question: Are the forms of diversity implied in our economic institutions consistent with humans' successful adaptation to change? Are our economic institutions sustainable?

\section{Definitions and Examples}

An Arrow Debreu market $\mathrm{E}=\left\{X, \Omega_{h}, u_{h}, h=1, \ldots, H\right\}$ has $H \geq 2$ traders, indexed $h=1, \ldots, H, N \geq 2$ commodities and consumption or trading space ${ }^{6} X=R_{+}^{N}$ or $X=R^{N}$; elsewhere I have considered the case where where instead of $R^{N}$ one has a Hilbert space of infinite dimension. ${ }^{7}$ The vector $\Omega_{h} \in R_{+}^{N}$ denotes trader $h$ 's property rights or initial endowment and $\Omega=\left(\sum_{h=1}^{H} \Omega_{h}\right)$ is the total endowment of the economy; when $X=R_{+}^{N}$, $\Omega>0 .{ }^{8}$ Traders may have zero endowments of some goods. Each trader $h$ has a continuous and convex preference represented by $u_{h}: X \rightarrow R$. This paper treats in a unified way general convex preferences where on every indifference surface for a given trader, the map $g(x)=D u(x) /\|D u(x)\|$ assigning to each vector the normalized utility gradient is either open or closed. Therefore either (i) all indifference surfaces contain no half lines (the map $g$ is open) or (ii) the normalized gradients to any closed set of indifferent vectors define a closed set (the map $g$ is closed). Some traders may have preferences of one type, and some of the other. Case (i) includes strictly convex preferences, and case (ii) linear preferences. All the assumptions and the results in this paper are ordinal; ${ }^{9}$ therefore without loss of generality one normalizes utilities so that for all $h, u_{h}(0)=0$ and $\sup _{\{\boldsymbol{x}: \boldsymbol{x} \in \boldsymbol{X}\}} u_{h}(x)=\infty$. Preferences are increasing, i.e. $x>y \Rightarrow u_{h}(x) \geq u_{h}(y)$. When $X=R_{+}^{N}$ either indifference surfaces of positive utility are contained in the interior

\footnotetext{
'Black's singlepeakedness condition restricts diversity and solves the problem proposed by Condorcet's [33] paradox of majority voting. ${ }^{5}$ Chichilnisky and Heal [32] established domain restrictions which are necessary and sufficient for the existence of social choice rules which satisfy the axioms of [12], [13]: contractibility of the space of preferences is necessary and sufficient for the existence of social choice rules. Although these works deal with somewhat different axioms, they all find the same type of solution: a restriction of individuals' diversity.

${ }^{6} R_{+}^{N}=\left\{\left(x_{1}, \ldots, x_{N}\right) \in R^{N}: \forall i, x_{i} \geq 0\right\}$.

${ }^{T}$ Chichilnisky and Heal [30] proved that limited arbitrage is necessary and sufficient for the existence of a competitive equilibrium in economies with infinitely many markets.

${ }^{8}$ If $x, y \in R^{N}, x \geq y \Leftrightarrow \forall i x_{i} \geq y_{i}, x>y \Leftrightarrow x \geq y$ and for some $i, x_{i}>y_{i}$, and $x>>y \Leftrightarrow \forall i, x_{i}>y_{i}$.

${ }^{9}$ Namely independent of the utility representations.
} 
of $X, R_{++}^{N}$, such as Cobb-Douglas utilities, or if an indifference surface of positive utility intersects a boundary ray, ${ }^{10}$ it does so transversally. ${ }^{11}$

Definition 1 A preference is uniformly non-satiated when it is represented by a utility $u_{h}$ with a bounded rate of increase, e.g. for smooth preferences: ${ }^{12} \exists \varepsilon, K>0: \forall x \in X$, $K>\left\|D u_{h}(x)\right\|>\varepsilon$.

Uniformly non-satiated preferences are rather common: for example, preferences represented by linear utilities are uniformly non-satiated..$^{13}$

Proposition 2 If a utility function $u_{h}: R^{N} \rightarrow R$ is uniformly non-satiated its indifference surfaces are within a uniform distance of each other, i.e. $\forall r, s \in R, \exists N(r, s) \in R$ such that $x \in u_{h}^{-1}(r) \Rightarrow \exists y \in u_{h}^{-1}(s)$ with $\|x-y\| \leq N(r, s)$.

Proof. This is immediate from the definition.

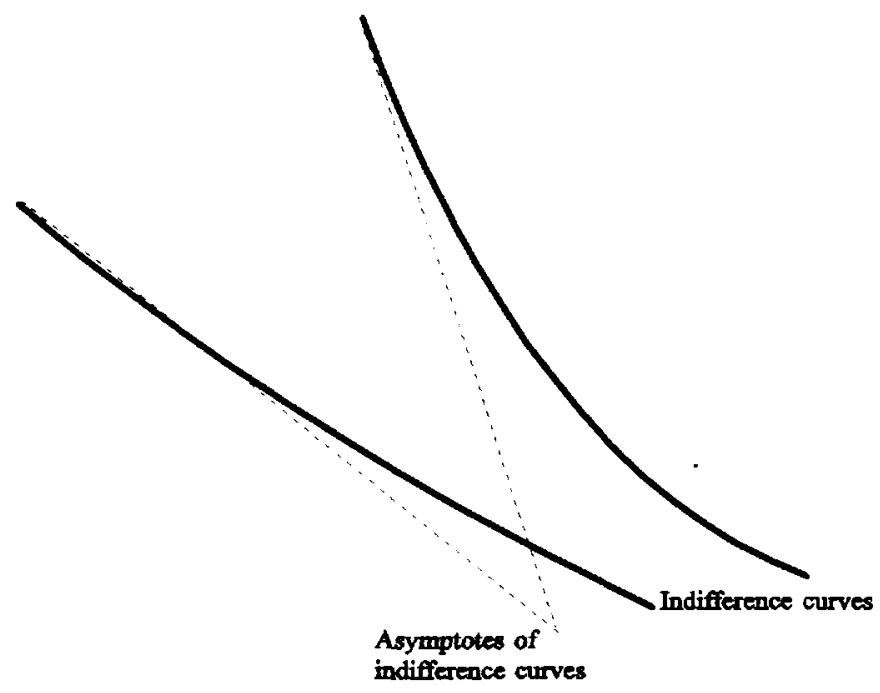

Figure 1. This preference is not uniformly nonsatiated because two indifference surfaces spread apart forever

\footnotetext{
${ }^{10} \mathrm{~A}$ boundary ray $r$ in $R_{+}^{N}$ is a set which consists of all the positive multiples of a vector $v \in \partial R_{+}^{N}$ : $r=\left\{w \in R_{+}^{N}: \exists \lambda>\mathbf{0}\right.$ s.t. $\left.w=\lambda v\right\}$

11 This means that if $x \in \partial R_{+}^{N}$ and $u(x)>0$, then $D u(x)$ is not orthogonal to $\partial R_{+}^{N}$ at $\lambda x, \forall \lambda \geq 0$. This condition includes strictly convex preferences, Cobb Douglas and CES preferences, many Leontieff preferences $u(x, y)=\min (a x, b y)$, preferences which are indifferent to one or more commodities, such as $u(x, y, z)=\sqrt{x+y}$, preferences with indifference surfaces which contain rays of $\partial R_{+}^{N}$ such as $u(x, y, z)=$ $x$, and preferences defined on a neighnorhood of the positive orthant or the whole space, and which are increasing along the boundaries, e.g. $u(x, y, z)=x+y+z$.

${ }^{12}$ Smoothness is used to simplify notation only: uniform non satiation requires no smoothness. When preferences admit no smooth utility representation, then one requires $\exists \varepsilon, K>0: \forall x, y, K\|x-y\|>\mid$ $u(y)-u(x) \mid$ and $\sup _{\|x\|<\delta}|u(y+x)| \geq \varepsilon \delta$.

${ }^{13}$ This includes Cobb-Douglas, constant elasticity of substitution (CES), preferences with indifference surfaces of positive consumption contained in the interior of $R_{+}^{N}$, linear preferences, piecewise linear preferences, most Leontief preferences, preferences with indifference surfaces which intersect the boundary of the positive orthant (Arrow and Hahn (1971)) and smooth utilities defined on a neighborhood of $X$ which are transversal to its boundary $\partial X$.
} 


\section{Assumption 1. When $X=R^{N}$, the preferences in the economy $\mathrm{E}$ are uniformly non-satiated.}

The space of feasible allocations is $\Upsilon=\left\{\left(x_{1}, \ldots, x_{H}\right) \in X^{H}: \sum_{h=1}^{H} x_{h}=\Omega\right\}$. The set of supports to individually rational affordable efficient resource allocations is:

$$
\begin{gathered}
S(\mathrm{E})=\left\{v \in R^{N}: \text { if }\left(x_{1} \ldots x_{H}\right) \in \Upsilon \text { with } u_{h}\left(x_{h}\right) \geq u_{h}\left(\Omega_{h}\right) \forall h=1, \ldots H,\right. \\
\left.\left\langle v \cdot x_{h}-\Omega_{h}\right\rangle=0, \text { and } u_{h}\left(z_{h}\right) \geq u_{h}\left(x_{h}\right) \text { then }\left\langle v, z_{h}-x_{h}\right\rangle \geq 0\right\} .
\end{gathered}
$$

The set of prices orthogonal to the endowments is ${ }^{14}$

$$
\mathbf{N}=\left\{v \in R_{+}^{N}-\{0\}: \exists h \text { with }\left\langle v, \Omega_{h}\right\rangle=0\right\} .
$$

The utility possibility set of the economy $E$ is the set of feasible and individually rational utility allocations:

$$
\begin{gathered}
U(\mathrm{E})=\left\{\left(V_{1}, \ldots, V_{H}\right): \forall h, V_{h}=u_{h}\left(x_{h}\right) \geq u_{h}\left(\Omega_{h}\right) \geq 0,\right. \\
\text { for some } \left.\left(x_{1}, \ldots, x_{H}\right) \in \Upsilon\right\} .
\end{gathered}
$$

The Pareto frontier of the economy $E$ is the set of feasible, individually rational and efficient utility allocations:

$$
P(\mathrm{E})=\{U \in U(\mathrm{E}): \sim \exists W \in U(\mathrm{E}): W>>U\}
$$

A competitive equilibrium of $\mathrm{E}$ consists of a price vector $p^{*} \in R_{+}^{N}$ and a feasible allocation $\left(x_{1}^{*} \ldots x_{H}^{*}\right) \in \Upsilon$ such that $x_{h}^{*}$ optimizes $u_{h}$ over the budget set $B_{h}\left(p^{*}\right)=\{x \in$ $\left.X:\left\langle x, p^{*}\right\rangle=\left\langle\Omega_{h}, p^{*}\right\rangle\right\}$.

\subsection{Global and Market Cones}

Two cases, $X=R^{N}$ and $X=R_{+}^{N}$, are considered separately.

- Consider first $X=R^{N}$.

Definition 3 For trader $h$ define the global cone of directions along which utility increases without bound:

$$
A_{h}\left(\Omega_{h}\right)=\left\{x \in X: \forall y \in X, \exists \lambda>0: u_{h}\left(\Omega_{h}+\lambda x\right)>u_{h}(y)\right\}
$$

This cone contains global information on the economy and is new in the literature. ${ }^{15}$ In ordinal terms, the rays of this cone intersect all indifference surfaces corresponding to bundles preferred by $u_{h}$ to $\Omega_{h}$. This cone and the part of its boundary along which utility never ceases to increase define:

$$
G_{h}\left(\Omega_{h}\right)=\left\{x \in X: \sim \exists \operatorname{Max}_{\lambda \geq 0} u_{h}\left(\Omega_{h}+\lambda x\right)\right\}
$$

\footnotetext{
${ }^{14} N$ is empty when $\forall h, \Omega_{h}>>0$.

${ }^{15}$ The global cone $A\left(\rho_{h}, \Omega_{h}\right)$ has points in common with Debreu's [34] "asymptotic cone" corresponding to the preferred set of $u_{h}$ at the initial endowment $\Omega_{h}$, in that along any of the rays of $A_{h}\left(\Omega_{h}\right)$ utility increases. Under Assumption 1, its closure $\bar{A}\left(\Omega_{h}\right)$, equals the "recession" cone used by Rockafeller in 1970 , but not generally: along the rays in $A\left(\Omega_{h}\right)$ utility increases beyond the utility level of any other vec tor in the space. This condition need not be satisfied by Debreu's asymptotic cones [34] or by "recession" cones. For example, for Leontief type preferences the recession cone through the endowment is the closure of the upper contour, which includes the indifference curve itself. By contrast, the global cone $A_{h}\left(\Omega_{h}\right)$ is the interior of the upper contour set. Related concepts appeared in Chichilnisky [10] [11]; otherwise there is no precedent in the literature for global cones.
} 
This cone treats all convex preferences in a unified way and under Assumption 1 it has a simple structure: when preferences have half lines in their indifferences $G_{h}\left(\Omega_{h}\right)$ equals $A_{h}\left(\Omega_{h}\right)$; when indifferences contain no half lines, then $G_{h}\left(\Omega_{h}\right)$ is its closure: it is therefore identical to the global cone defined Chichilnisky 1995, p. $85,(4) .{ }^{16}$

Definition 4 The market cone of trader $h$ is

$$
D_{h}\left(\Omega_{h}\right)=\left\{z \in X: \forall y \in G_{h}\left(\Omega_{h}\right),\langle z, y\rangle>0\right\}
$$

$D_{h}$ is the cone of prices assigning strictly positive value to all directions of net trades leading to eventually increasing utility. This is a convex cone.

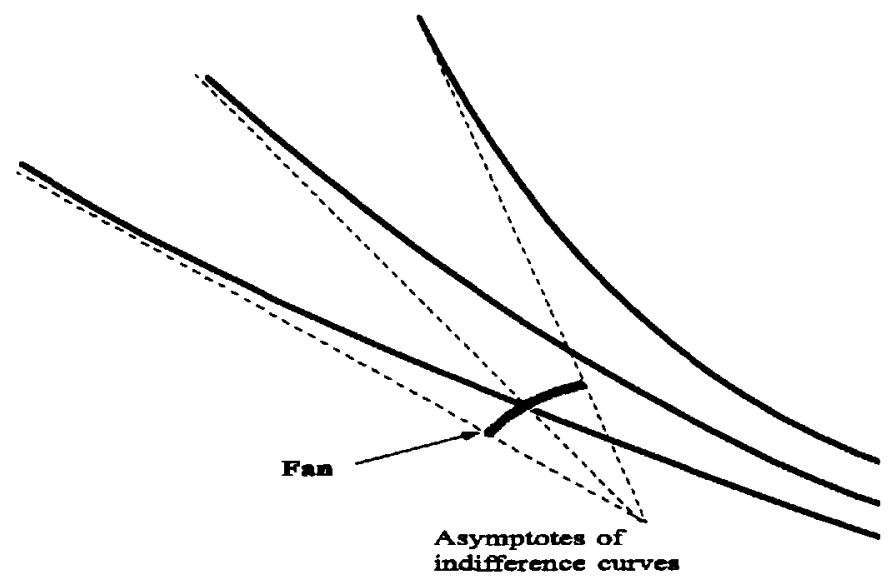

Figure 2. This preference has a 'fan' of directions along which the utility values reach a bound. Assumption 1 is not satisfied. All the directions in the fan are in the recession cone but none in the global cone $\mathrm{G}_{h}$ or in the cone $A_{h}$.

The following proposition establishes the structure of the global cones, and is used in proving the connection between limited arbitrage, equilibrium and the core:

Proposition 5 If the function $u_{h}: R^{N} \rightarrow R$ is uniformly non-satiated: (i) The interior of the global cone is

$$
\begin{aligned}
G_{h}^{o}\left(\Omega_{h}\right) & =A_{h}^{o}\left(\Omega_{h}\right) \\
& =\left\{z \in G_{h}\left(\Omega_{h}\right): \lim _{\lambda \rightarrow \infty} u_{h}\left(\Omega_{h}+\lambda z\right)=\infty\right\} \neq \emptyset
\end{aligned}
$$

(ii) The boundary of the cone $G_{h}\left(\Omega_{h}\right), \partial G_{h}\left(\Omega_{h}\right)$, contains (a) those directions along which utility increases towards a bounded value that is never reached:

$$
B_{h}\left(\Omega_{h}\right)=\left\{z \in \partial A_{h}\left(\Omega_{h}\right): \forall \lambda>0, u_{h}\left(\Omega_{h}+\lambda z\right) \neq \lim _{\lambda \rightarrow \infty} u_{h}\left(\Omega_{h}+\lambda z\right)<\infty\right\}
$$

and (b) those directions along which the utility eventually achieves a constant value:

$$
C_{h}\left(\Omega_{h}\right)=\left\{z \in \partial A_{h}\left(\Omega_{h}\right): \exists N: \lambda, \mu>N \Rightarrow u_{h}\left(\Omega_{h}+\lambda z\right)=u_{h}\left(\Omega_{h}+\mu z\right)\right\},
$$

\footnotetext{
${ }^{16}$ The cone $G_{h}\left(\Omega_{h}\right)$ is that in Chichilnisky [18] under Assumption 1, and it appears also in Monteiro Page and Wooders [50] which is a comment on Chichilnisky [18].
} 
(iii) the interior of the global cone, its boundary and its closure and the cones $G_{h}$ and $D_{h}$ are uniform across all vectors in the space, i.e. $\forall \Omega, \Lambda \in X$ :

$$
\begin{aligned}
G_{h}^{o}(\Omega) & =G_{h}^{o}(\Lambda)=A_{h}^{o} \\
B_{h}(\Omega) \cup C_{h}(\Omega) & \subset \partial G_{h}(\Omega)=\partial G_{h}(\Lambda)=\partial G_{h} \\
G_{h}(\Omega) & =G_{h}(\Lambda)=G_{h}, D_{h}(\Omega)=D_{h}(\Lambda)=D_{h}
\end{aligned}
$$

and in particular

$$
\bar{G}_{h}(\Omega)=\bar{G}_{h}(\Lambda)=\bar{G}_{h}
$$

(iv) For general non-satiated preferences $G_{h}\left(\Omega_{h}\right)$ and $D_{h}\left(\Omega_{h}\right)$ may not be uniform. Proof. See [26], Proposition 3, Appendix.

- Consider next the case: $X=R_{+}^{N}$

Definition 6 The market cone of trader $h$ is:

$$
\begin{aligned}
D_{h}^{+}\left(\Omega_{h}\right) & =D_{h}\left(\Omega_{h}\right) \cap S(\mathrm{E}) \text { if } S(\mathrm{E}) \subset \mathrm{N}, \\
& =D_{h}\left(\Omega_{h}\right) \text { otherwise. }
\end{aligned}
$$

where $S(\mathrm{E})$ and $\mathrm{N}$ are defined in (1) and (2). ${ }^{17}$

There is no analog to Proposition 2 when $X=R_{+}^{N}$; indeed, when $X=R_{+}^{N}$ the market cones $D_{h}^{+}\left(\Omega_{h}\right)$ typically vary with the initial endowments.

Proposition 7 When $X=R_{+}^{N}$ and an indifference surface of $u_{h}$ corresponding to a positive consumption bundle $x>0$ intersects a boundary ray $r \subset \partial X$, then $r \in G_{h}(0) .{ }^{32}$

Proof. Recall that we assumed $u_{h}(0)=0$, and that the preference's indifference surfaces of positive utility are either (a) contained in the interior of $R_{+}^{N}, R_{++}^{N}$, or (b) they intersect a boundary ray $r$ of $R_{+}^{N}$ and do so transversally. In case (a) the proposition is satisfied trivially, because no indifference surface of strictly positive value ever intersects the boundary of $R_{+}^{N}$. In case (b) the proposition follows immediately from the definition of transversality. Observe that it is possible that $\sup _{x \in r}\left(u_{h}(x)\right)<\infty$.

Definition 8 The core of the economy $E$ is the set of allocations which no coalition can improve upon within its own endouments:

$$
\begin{gathered}
C(\mathrm{E})=\left\{\left(x_{1}, \ldots, x_{H}\right) \in R^{N \times H}: \sum_{h}\left(x_{h}-\Omega_{h}\right)=0 \text { and } \sim J \subset\{1, \ldots, H\}:\right. \\
\sum_{j \in J}\left(y_{j}-\Omega_{j}\right)=0, \forall j \in J, u_{j}\left(y_{j}\right) \geq u_{j}\left(x_{j}\right), \\
\text { and } \left.\exists j \in J: u_{j}\left(y_{j}\right)>u_{j}\left(x_{j}\right)\right\} .
\end{gathered}
$$

\section{Limited Arbitrage: Definition and Examples}

This section provides the definition of limited arbitrage. It gives an intuitive interpretation for limited arbitrage in terms of gains from trade, and contrasts limited arbitrage with the arbitrage concept used in financial markets. It provides examples of economies with and without limited arbitrage.

\footnotetext{
${ }^{17}$ The market cone $D_{h}^{+}$is the whole consumption set $X=R^{N+}$ when $S(E)$ has a vector assigning strictly positive income to all individuals. If some trader has zero income, then this trader must have a boundary endow ment.
} 
Definition 9 When $X=R^{N}$, E satisfies limited arbitrage when

$$
\text { (LA) } \bigcap_{h=1}^{H} D_{h} \neq \emptyset .
$$

Definition 10 When $X=R_{+}^{N}$, E satisfies limited arbitrage when

$$
\left(L A^{+}\right) \bigcap_{h=1}^{H} D_{h}^{+}\left(\Omega_{h}\right) \neq \emptyset .
$$

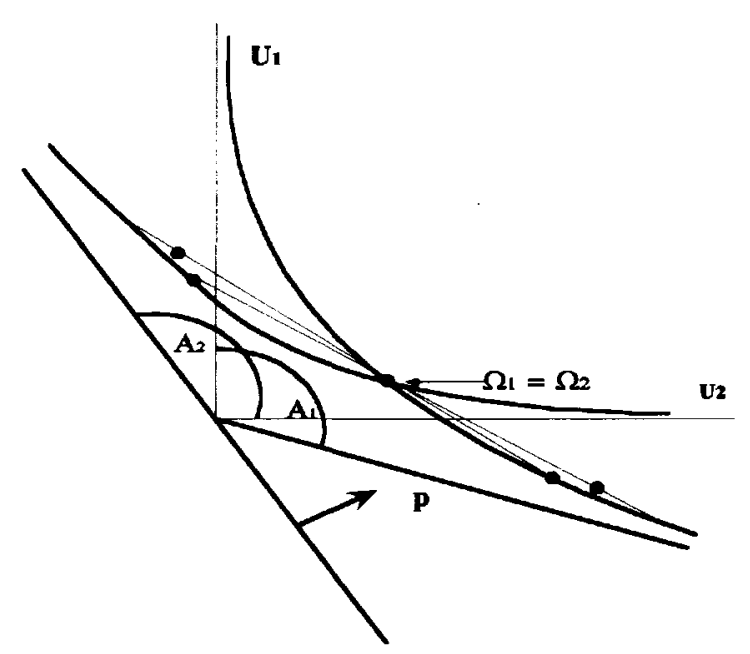

Figure 3. Limited arbitrage is satisfied: feasible allocations lead to bounded utility increases.

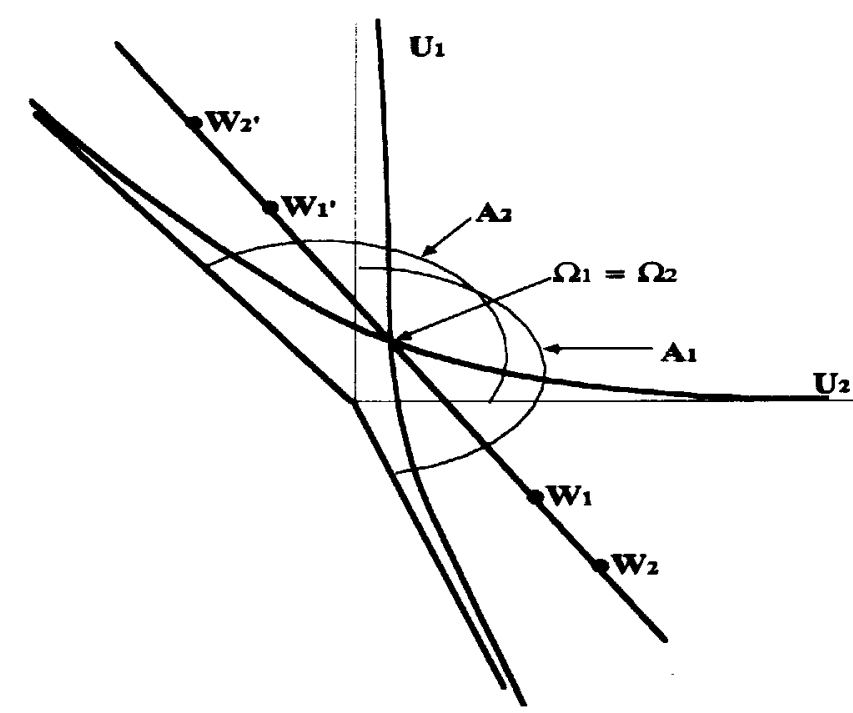

Figure 4. Limited arbitrage is not satisfied: there exist a feasible unbounded sequence of allocations, $\left(W_{1}, W_{1}^{\prime}\right),\left(W_{2}, W_{2}^{\prime}\right), \ldots$, along which both traders' utility never ceases to increase. 


\subsection{Interpretation of Limited Arbitrage as Bounded Gains From Trade when $X=R^{N}$}

Gains from trade are defined by:

$$
\begin{gathered}
\mathrm{G}(\mathrm{E})=\sup \left\{\sum_{h=1}^{H}\left(u_{h}\left(x_{h}\right)-u_{h}\left(\Omega_{h}\right)\right\},\right. \text { where } \\
\sum_{h=1}^{H}\left(x_{h}-\Omega_{h}\right)=0, \text { and } \forall h, u_{h}\left(x_{h}\right) \geq u_{h}\left(\Omega_{h}\right) \geq 0 .
\end{gathered}
$$

The Proposition below applies to preferences where the normals to a closed set of indifferent vectors defines a closed set, case (ii); the Corollary following it applies both to case (i) and (ii):

Proposition 11 In case (ii), the economy $\mathrm{E}$ satisfies limited arbitrage if and only if gains from trade are bounded i.e. if and only if $1^{8}$

$$
\mathrm{G}(\mathrm{E})<\infty
$$

For a proof see Theorem 1 proved in the Appendix of [26]; its sufficiency part is valid for all preferences satisfying Assumption 1, case (i) or case (ii), so that:

Corollary 12 For all economies with uniformly non-satiated preferences, limited arbitrage implies bounded gains from trade. ${ }^{19}$

\subsection{Examples of markets with and without limited arbitrage}

Example 13 Figures 3 and 4 above illustrate an economy with two traders trading in $X=R^{2} ;$ in Figure 3 the market cones intersect and the economy has limited arbitrage. In Figure 4 the market cones do not intersect and the economy does not have limited arbitrage. Figure 5 below illustrates three traders trading in $X=R^{3}$; each two market cones intersect, but the three market cones do not intersect, and the economy violates limited arbitrage. This figure illustrates the fact that the union of the market cones may fail to be contractible: indeed, this failure corresponds to the failure of the market cones to intersect, as proven in Chichilnisky [19].

\footnotetext{
${ }^{18}$ The expression $G(E)<\infty$ holds when $\forall h, \sup _{\{x: x \in X} ; u_{h}(x)=\infty$; it must be replaced by $G(E)<\sup _{\left\{x: x \in X^{H}: \sum x_{h}=\Omega, u_{h}\left(x_{h}\right) \geq u_{h}\left(\Omega_{h}\right)\right\}}\left(\sum_{h=1}^{H} u_{h}\left(x_{h}\right)-u_{h}\left(\Omega_{h}\right)\right)-k$, for some positive $k$, when and $\sup _{\{\boldsymbol{x}: \boldsymbol{x} \in X\}} u_{h}(x)<\infty$.

${ }^{19}$ The simplest illustration of the link between limited arbitrage and no-arbitrage is an economy $E$ where the traders' initial endowments are zero, $\Omega_{h}=0$ for $h=1,2$, and the set of gradients to indifference surfaces are closed. Here no-arbitrage at the initial endowments means that there are no trades which could increase the traders' utilities at zero cost: gains from trade in $E$ are zero. By contrast, $E$ has limited arbitrage when no trader can increase utility beyond a given bound at zero cost: gains from trade are bounded.

In brief: no-arbitrage requires that there should be no gains from trade at zero cost while limited arbitrage requires that there should be only bounded utility arbitrage or limited gains from trade. In linear economies, limited arbitrage "collapses" into no-arbitrage.
} 


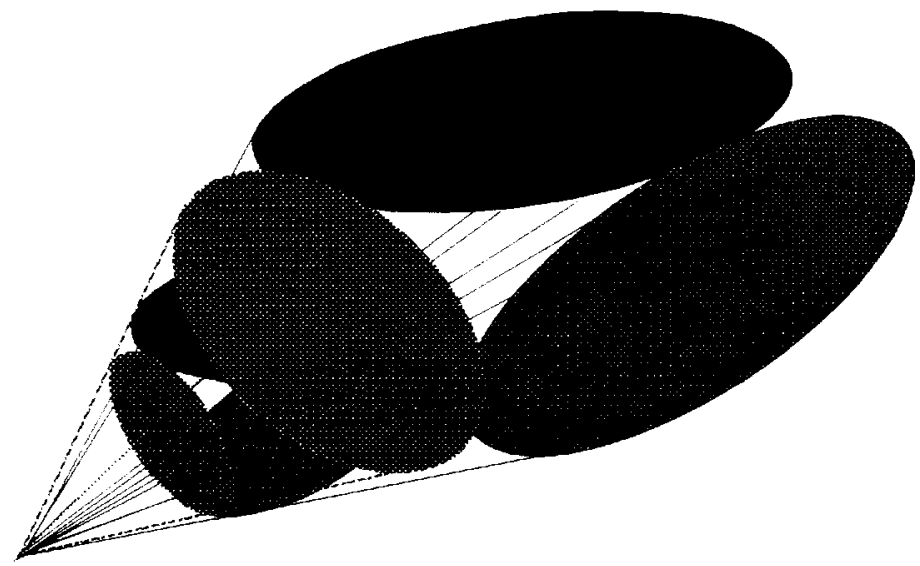

Figure 5. Three traders in $\mathrm{R}^{3}$. Every two traders's subeconomy has limited arbitrage but the whole economy does not.

Example 14 When the consumption set is $X=R_{+}^{N}$, limited arbitrage is always satisfied if all indifference surfaces through positive consumption bundles are contained in the interior of $X, R_{-+}^{N}$. Examples of such preferences are those given by Cobb-Douglas utilities, or by utilities with constant elasticity of substitution (CES) with elasticity of substitution $\sigma<1$. This is because all such preferences have as global cone the positive orthant (or its closure), and therefore their market cones always intersect. These preferences are very similar to each other on choices involving large utility levels: this is a form of similarity of preferences. Economies where the individuals' initial endowments are strictly interior to the consumption set $X$ always satisfy the limited arbitrage condition in the case $X=R_{+}^{N}$, since in this case $\forall h, R_{++}^{N} \subset D_{h}^{+}\left(\Omega_{h}\right)$ for all $h=1, \ldots, H$.

Example 15 When $X=R_{+}^{N}$ the limited arbitrage condition may fail to be satisfied when some trader's endowment vector $\Omega_{h}$ is in the boundary of the consumption space, $\partial R_{+}^{N}$, and at all supporting prices in $S(\mathrm{E})$ some trader has zero income, i.e. when $\forall p \in S(\mathrm{E})$ $\exists$ such that $\left\langle p, \Omega_{h}\right\rangle=0$. In this case, $S(\mathrm{E}) \subset \mathrm{N}$. This case is illustrated in Figure 6 below; it is a rather general case which may occur in economies with many individuals and with many commodities. When all individuals have positive income at some price $p \in S(\mathrm{E})$, then limited arbitrage is always satisfied since by definition in this case $\forall$, $=R_{++}^{N} \subset D_{h}^{+}\left(\Omega_{i}\right)$ for all $h=1, \ldots, H$.

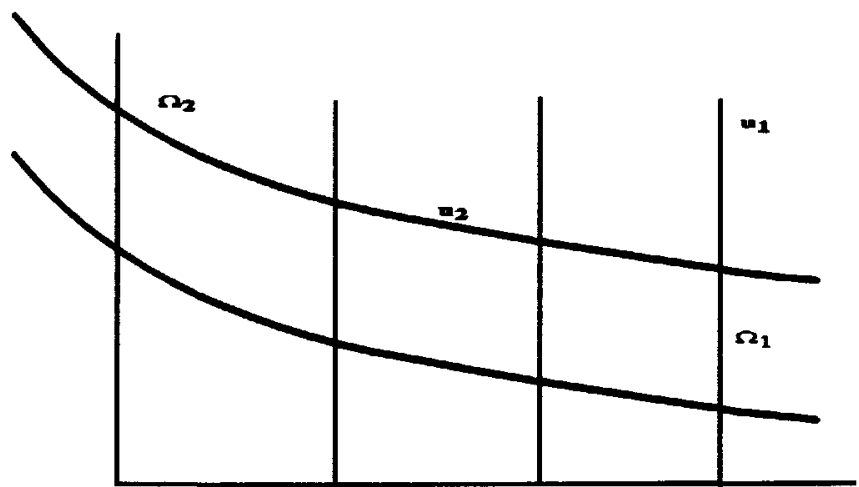

Figure 6. Limited arbitrage fails. Trader two only owns one good, to which the first trader is indifferent. 
Example 16 A competitive equilibrium may exist even when some traders have zero income, showing that Arrow's "resource relatedness" condition [3] is sufficient but not necessary for existence of an equilibrium. Figure 7 below illustrates an economy where at all supporting prices some trader has zero income: $\forall p \in S(\mathrm{E}) \exists h$ such that $\left\langle p, \Omega_{h}\right\rangle=0$, i.e. $S(\mathrm{E}) \subset \mathrm{N}$; in this economy, however, limited arbitrage is satisfied so that a competitive equilibrium exists. The initial allocation and a price vector assigning value zero to the second good defines such an equilibrium.

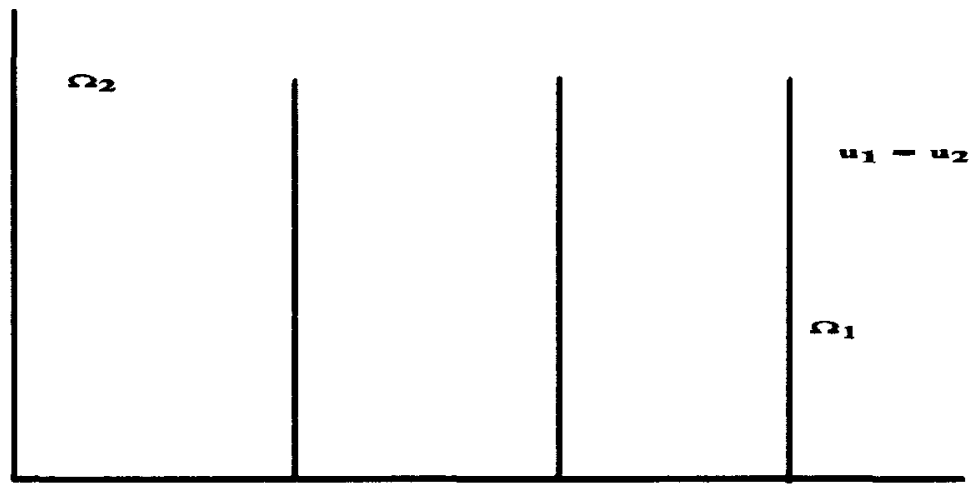

Figure 7. Equilibrium exists even when one trader has zero income

\section{Limited Arbitrage and the Compactness of the Pareto Frontier}

The Pareto frontier $P(E)$ is the set of feasible, efficient and individually rational utility allocations. With $H$ traders it is a subset of $R_{+}^{H}$. Proving the boundedness and closedness of the Pareto frontier is a crucial step in establishing the existence of a competitive equilibrium and the non-emptiness of the core. The main theorem of this section shows that limited arbitrage is necessary and sufficient for this.

There is a novel feature of the results which are presented here, a feature which is shared which those that were previously established in Chichilnisky [18], [20], [30], [26], and Chichilnisky and Heal [29], [30]. It starts from the observation that the compactness of the Pareto frontier need not imply the compactness of the set of feasible commodity allocations. Indeed, such boundedness is not used in this paper, nor was it used in the results of Chichilnisky [18], [20], [30], [26] and Chichilnisky and Heal [30]: these are the first results in the literature proving the existence of equilibrium and the non-emptiness of the core in economies where limited arbitrage holds and the set of feasible and individually rational allocations is generally unbounded.

Example 17 Figure 8 shows that the Pareto frontier may fail to be closed even in finite dimensional models, provided the consumption set is the whole Euclidean space. It shows two traders with indifference curves having the line $y=-x$ as asymptote. Consumption sets are the whole space and feasible allocations are those which sum to zero. Utility functions are $u_{i}=x_{i}+y_{i} \pm e^{-\left(x_{i}-y_{i}\right)}, i=1,2$. Limited arbitrage rules out such cases. Another example is a two-agent economy where both agents have linear preferences: if the preferences are different the set of feasible utility allocations is unbounded. Of course, limited arbitrage rules out such situations. 


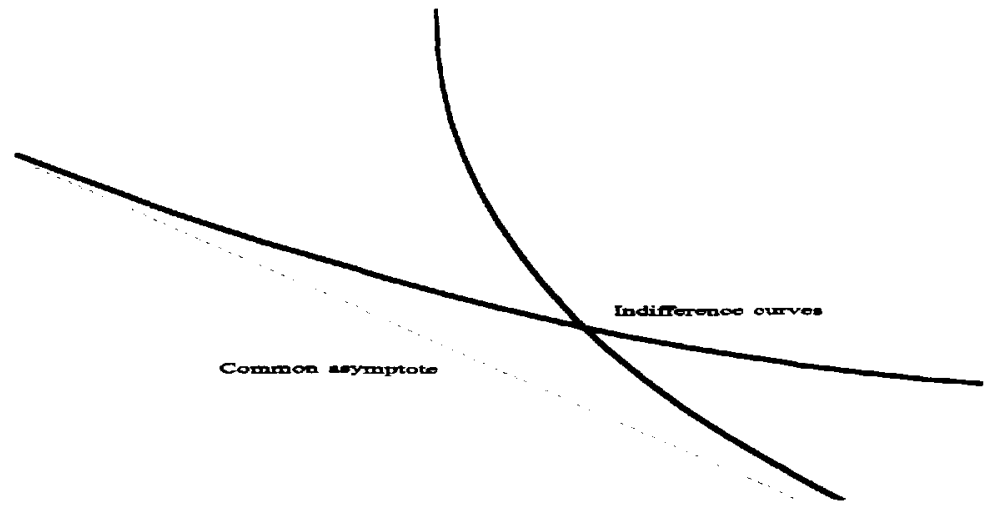

Figure 8. The Pareto frontier may fail to be closed even in finite dimensions

Theorem 18 Consider an economy $\mathrm{E}$ as defined in Section 1. Then limited arbitrage is necessary and sufficient for the compactness of the Pareto frontier.

See the Proof of Theorem 1 in the Appendix of [26].

\section{Competitive Equilibrium and Limited Arbitrage}

This section links the existence of a competitive equilibrium with limited arbitrage. The result is that limited arbitrage is simultaneously necessary and sufficient for the existence of a competitive equilibrium.

The result presented below was established first in Chichilnisky [18], [20], [21], [26], [30] for preferences which are either all in case (i) e.g. strictly convex, or in case (ii), e.g. they have indifference surfaces with a closed set of gradient directions. The result presented here extends these earlier results in that it deals in a unified way with non-satiated convex preferences: in the same economy there may be a mixture of preferences of type (i) and (ii)..$^{20}$

The condition of limited arbitrage need not be tested on all traders simultaneously: in the case of $R^{N}$, it needs only be satisfied on subeconomies with no more traders than the number of commodities in the economy, $N$, plus one.

Definition $19 A k$-trader sub-economy of $\mathrm{E}$ is an economy $F$ consisting of a subset of $k \leq H$ traders in $\mathrm{E}$, each with the endowments and preferences as in $\mathrm{E}: \mathrm{F}=\left\{X, u_{h}, \Omega_{h}, h \in\right.$ $J \subset\{1, \ldots, H\}$, cardinality $(J)=k \leq H\}$.

Theorem 20 The following four properties of an economy $E$ with trading space $R^{N}$ are equivalent:

(i) E has a competitive equilibrium

(ii) Every sub economy of $\mathrm{E}$ with at most $N+1$ traders has a competitive equilibrium

\footnotetext{
${ }^{20}$ The results on equilibrium in this paper originated from a theorem in Chichilnisky and Heal [29] as paper which was submitted for publication in 1984, nine years before it appeared in print: these dates are recorded in the printed version [32]. Chichilnisky and Heal [29] [32] provide a no-arbitrage condition and prove that it is sufficient for the existence of a competitive equilibrium and the compactness of the utility set in Arrow Debreu economies with or without short sales, with infinitely or finitely many markets and with general preferences which are convex or strictly convex. Subsequent to [29] Werner [56] proved existence results for markets with short sales, relying on a no-arbitrage condition defined from recession cones. For Hart-type models which are incomplete as they lack futures markets and which do not have the generality of the Arrow Debreu model, Page [53] uses Werner's no-arbitrage condition to prove existence of a market equilibrium. The equilibria in Hart-type models are generally inefficient, and their allocations are not in the Pareto frontier.
} 
(iii) E has limited arbitrage

(iv) E has limited arbitrage for any subset of traders with no more that $N+1$ members.

Proof. See the proof of Theorem 2 in the Appendix of [26] for (i) $\Leftrightarrow$ (iii) and (ii) $\Leftrightarrow$ (iv). That (iii) $\Leftrightarrow$ (iv) follows from Helley's theorem, which is a corollary in Chichilnisky [19]: Consider a family $\left\{U_{i}\right\}_{i=1 \ldots H}$ of convex sets in $R^{N} . H, N \geq 1$. Then

$$
\bigcap_{i=1}^{H} U_{i} \neq \emptyset \text { if and only if } \bigcap_{j \in J} U_{i} \neq \emptyset
$$

for any subset of indices $J \subset\{1 \ldots H\}$ having at most $N+1$ elements.

In particular, an economy $E$ as defined in Section 2 satisfies limited arbitrage, if and only if it satisfies limited arbitrage for any subset of $k=N+1$ traders, where $N$ is the number of commodities in the economy $E$.

\section{Limited Arbitrage, Equilibrium and The Core}

The following is a corollary of Theorem 2:

Theorem 21 Consider an economy $\mathrm{E}=\left\{X, u_{h}, \Omega_{h}, h=1, \ldots, H\right\}$, where $H \geq 2, X=R^{N}$ and $N \geq 1$, or $X$ is a Hilbert space $H$. Then the following four properties are equivalent:

(i) The economy $\mathrm{E}$ has limited arbitrage

(ii) The economy $\mathrm{E}$ has a core

(iii) The economy $\mathrm{E}$ has a competitive equilibrium

(iv) When $X=R^{N}$, every subeconomy of $E$ with at most $N+1$ trades has a competitive equilibrium and a core.

Proof. For a proof of (i) $\Leftrightarrow($ ii $) \Leftrightarrow$ (iii) see [21], which contains also a discussion of the literature. ${ }^{21}$

Finally I establish (iv) $\Leftrightarrow$ (i): this follows directly from the fact that an economy has limited arbitrage if and only if every subeconomy of at most $N+1$ traders has limited arbitrage, Theorem 9 in the Appendix.

\section{Limited Arbitrage and Social Choice}

Limited arbitrage is also crucial for achieving resource allocation via social choice. Two main approaches to social choice are studied here. One is Arrow's: his axioms of social choice require that the social choice rule $\Phi$ be non-dictatorial, independent of irrelevant alternatives, and satisfy a Pareto condition Arrow [2]. A second approach requires, instead, that the rule $\Phi$ be continuous, anonymous, and respect unanimity, Chichilnisky [12], [13]. Both approaches have led to corresponding impossibility results [2], [12], [13]. Though the two sets of axioms are quite different, it has been shown recently that the impossibility results which emerge from them are equivalent, see Baryshnikov [7]. Furthermore, as is shown below, limited arbitrage is closely connected with both sets of axioms. Economies which satisfy limited arbitrage admit social choice rules with either

\footnotetext{
21 This result was presented at the January 3-5, 1994 Annual Meetings of the Econometric Society in Boston. Page acted as referee for [24]. Subsequently I received a working paper by Page and Wooders on a related topic; see Monteiro Page and Wooders [50] which is a comment on Chichilnisky [19], [22] and [24], and the response in Chichilnisky [26].
} 
set of axioms. Therefore, in a well defined sense, the social choice problem can only be solved in those economies which satisfy limited arbitrage.

How do we allocate resources by social choice? Social choice rules assign a social preference $\Phi\left(u_{1} \ldots u_{H}\right)$ to each list $\left(u_{1} \ldots u_{H}\right)$ of individual preferences of an economy $E .{ }^{22}$ The social preference ranks allocations in $R^{N \times H}$, and allows one to select an optimal feasible allocation. This is the resource allocation obtained via social choice.

The procedure requires, of course, that a social choice rule $\Phi$ exists: the role of limited arbitrage is important because it ensures existence. This will be established below. I prove here that limited arbitrage is necessary and sufficient for resolving Arrow's paradox when the domain of individual preferences are those in the economy, and the choices are those feasible allocations which give large utility value. ${ }^{23}$

Limited arbitrage provides a restriction on the relationship between individual preferences under which social choice rules exist. A brief background on the matter of preference diversity follows.

Arrow's impossibility theorem established that in general a social choice rule $\Phi$ does not exist: the problem of social choice has no solution unless individual preferences are restricted. Duncan Black [8] established that the "single peakedness" of preferences is a sufficient restriction to obtain majority rules. Using different axioms, Chichilnisky [12], [13] established also that a social choice rule $\Phi$ does not generally exist; subsequently Chichilnisky and Heal [29] established a necessary and sufficient restriction for the resolution of the social choice paradox: the contractibility of the space of preferences. Contractibility can be interpreted as a limitation on preference diversity, Heal [43]. In all cases, therefore, the problem of social choice is resolved by restricting the diversity of individual preferences. The main result in this section is that the restriction on individual preferences required to solve the problem is precisely limited arbitrage. The connection between limited arbitrage and contractibility is discussed below.

The section is organized as follows. First I show in Proposition 6 that the economy E satisfies limited arbitrage if and only if it contains no Condorcet cycles on choices of large utility values. Condorcet cycles are the building blocks of Arrow's impossibility theorem, and are at the root of the social choice problem. On the basis of Proposition 6, I prove in Theorem 9 that limited arbitrage is necessary and sufficient for resolving Arrow's paradox on allocations of large utility values.

Definition 22 A Condorcet cycle is a collection of three preferences over a choice set $X$, represented by three utilities $u_{i}: X \rightarrow R, i=1,2,3$, and three choices $\alpha, \beta, \gamma$ within a feasible set $Y \subset X$ such that $u_{1}(\alpha)>u_{1}(\beta)>u_{1}(\gamma), u_{2}(\gamma)>u_{2}(\alpha)>u_{2}(\beta)$ and $u_{3}(\beta)>u_{3}(\gamma)>u_{3}(\alpha)$.

Within an economy with finite resources $\Omega>>$, the social choice problem is about the choice between allocations of these resources. Choices are in $X=R^{N \times H}$. An allocation $\left(x_{1} \ldots x_{H}\right) \in R^{N \times H}$ is feasible if $\sum_{i} x_{i}-\Omega=0$. Consider an economy $\mathrm{E}$ as defined in Section 2. Preferences over private consumption are increasing, $u_{h}(x)>u_{h}(y)$ if $x>y \in R^{N}$, utilities are uniformly non-satiated (Assumption 1), and indifference surfaces which are not bounded below have a closed set of gradients, so that $G_{h}=A_{h}$. While the preferences in $E$ are defined over private consumption, they naturally define preference over allocations, as follows: define $u_{h}\left(x_{1} \ldots x_{H}\right) \geq u_{h}\left(y_{i} \ldots y_{H}\right) \Leftrightarrow u_{h}\left(x_{h}\right) \geq u_{h}\left(y_{h}\right)$. Thus the preferences in the economy $E$ induce naturally preferences over the feasible allocations in $E$.

\footnotetext{
${ }^{22}$ In the economy $E$ the traders' preferences are defined over private consumption $u_{i}: R^{N} \rightarrow R$, but they define automatic ally preferences over allocations in $R^{N \times H}: u_{i}\left(x_{1} \ldots x_{H}\right) \geq u_{i}\left(y_{1} \ldots y_{H}\right) \Leftrightarrow u_{i}\left(x_{i}\right) \geq u_{i}\left(y_{i}\right)$.

${ }^{23}$ The concept of "large utility values" is purely ordinal; it is defined relative to the maximum utility value achieved by a utility representation.
} 
Definition 23 The family of preferences $\left\{u_{1} \ldots u_{H}\right\}, u_{h}: R^{N} \rightarrow R$ of an economy $\mathrm{E}$ has a Condorcet cycle of size $k$ if for every three preferences $u_{1}^{k}, u_{2}^{k}, u_{3}^{k} \in\left\{u_{1} \ldots u_{H}\right\}$ there exists three feasibie allocations $\alpha^{k}=\left(\alpha_{1}^{k}, \alpha_{2}^{k}, \alpha_{3}^{k}\right) \in X^{3 \times H} \subset R^{3 \times N \times H} ; \beta^{k}=\left(\beta_{1}^{k}, \beta_{2}^{k}, \beta_{3}^{k}\right)$ and $\gamma^{k}=\left(\gamma_{1}^{k}, \gamma_{2}^{k}, \gamma_{3}^{k}\right)$ which define a Condorcet cycle, and such that each trader $h=1, \ldots, H$, achieves at least a utility level $k$ at each choice:

$$
\min _{h=1, \ldots, H}\left\{\left[u_{h}^{k}\left(\alpha_{h}^{k}\right), u_{h}^{k}\left(\beta_{h}^{k}\right), u_{h}\left(\gamma_{h}^{k}\right)\right]\right\}>k .
$$

The following shows that limited arbitrage eliminates Condorcet cycles on matters of great importance, namely on those with utility level approaching the supremum of the utilities. which for simplicity and without loss of generality we have assumed to be $\infty$ :

Proposition 24 Let $\mathrm{E}$ be a market economy with short sales $\left(X=R^{N}\right)$ and $H \geq 3$ traders. Then $\mathrm{E}$ has social diversity if and only if its traders' preferences have Condorcet cycles of every size. Equivalently, E has limited arbitrage if and only for some $k>0$, the traders' preferences have no Condorcet cycles of size larger than $k$.

Proof. Consider an economy with Condorcet cycles of all sizes. For each $k>0$, there exists three allocations denoted $\left(\alpha^{k}, \beta^{k}, \gamma^{k}\right) \in R^{3 \times N \times H}$ and three traders $u_{k_{1}}^{k}, u_{k_{2}}^{k}, u_{k_{3}}^{k} \subset$ $\left\{u_{1} \ldots u_{H}\right\}$ which define a Condorcet triple of size $k$. By definition, for every $k$, each of the three allocations is feasible, for example, $\alpha^{k}=\left(\alpha_{1}^{k}, \ldots, \alpha_{H}^{k}\right) \in R^{N \times H}$, and $\sum_{i=1}^{H}\left(\alpha_{i}^{k}\right)=0$. Furthermore $\min _{h=1, \ldots, H}\left\{\left[u_{h}^{k}\left(\alpha_{h}^{k}\right), u_{h}^{k}\left(\beta_{h}^{k}\right), u_{h}\left(\gamma_{h}^{k}\right)\right]\right\}>k$, so that e.g. $\lim _{k \rightarrow \infty}\left(u_{h}\left(\alpha_{h}^{k}\right)\right)=$ $\infty$. There exist therefore a sequence of allocations $\left(\theta^{k}\right)_{k=1,2 \ldots}=\left(\theta_{1}^{k}, \ldots, \theta_{H}^{k}\right)_{k=1,2 \ldots}$ such that $\forall k, \sum_{h=1}^{H} \theta_{h}^{k}=0$ and $\forall h \sup _{k \rightarrow \infty}\left(\inf _{i=1,2,3}\left(u_{h}\left(\theta_{h}^{k}\right)\right)=\infty\right.$. This implies that $\mathrm{E}$ has unbounded gains from trade, which contradicts Proposition 3 . Therefore $E$ cannot have Condorcet cycles of every size.

Conversely, if $\mathrm{E}$ has no limited arbitrage, for any $k>0$, there exist a feasible allocation $\left(a_{1}^{k}, a_{2}^{k}, \ldots, a_{H}^{k}\right)$, such that $\sum_{h=1}^{H} a_{h}^{k} \leq 0$, and $\forall h, u_{h}\left(a_{h}^{k}\right) \geq k$. For each integer $k>0$, and for a small enough $\varepsilon>0$ define now the vector $\Delta=(\varepsilon, \ldots, \varepsilon) \in R_{+}^{N}$ and the following three allocations: $\alpha^{k}=\left(k a_{1}^{k}, k a_{2}^{k}-2 \Delta, k a_{3}^{k}+2 \Delta, k a_{4}^{k}, \ldots, k a_{H}^{k}\right), \beta^{k}=\left(k a_{1}^{k}-\Delta, k a_{2}^{k}, k a_{3}^{k}+\right.$ $\left.\Delta, k a_{4}^{k}, \ldots, k a_{H}^{k}\right)$ and $\gamma^{k}=\left(k a_{1}^{k}-2 \Delta, k a_{2}^{k}-\Delta, k a_{3}^{k}+3 \Delta, k a_{4}^{k}, . . k a_{H}^{k}\right)$. Each allocation is feasible, e.g. $k a_{1}^{k}+k a_{2}^{k}-2 \Delta+k a_{3}^{k}+2 \Delta+k a_{4}^{k}+\ldots+k a_{H}^{k}=k\left(\sum_{h=1}^{H} a_{h}^{k}\right) \leq 0$. Furthermore for each $k>0$ sufficiently large, the three allocations $\alpha^{k}, \beta^{k}, \gamma^{k}$ and the traders $h=1,2,3$, define a Condorcet cycle of size $k$ : all traders except for $1,2,3$, are indifferent between the three allocations and they reach a utility value at least $k$, while trader 1 prefers $\alpha^{k}$ to $\beta^{k}$ to $\gamma^{k}$, trader 3 prefers $\gamma^{k}$ to $\alpha^{k}$ to $\beta^{k}$, and trader 2 prefers $\beta^{k}$ to $\gamma^{k}$ to $\alpha^{k}$. Observe that this construction can be made for any three traders within the set $\{1,2, \ldots, H\}$. This completes the proof.

The next result uses Proposition 5 to establish the connection between limited arbitrage and Arrow's theorem. Consider Arrow's three axioms: Pareto, independence of irrelevant alternatives, and non-dictatorship. The social choice problem is to find a social choice rule $\Phi: P^{j} \rightarrow P$ from individual to social preferences satisfying Arrow's three axioms; the domain for the rule $\Phi$ are profiles of individual preferences over allocations of the economy E: $\Phi: P^{j} \rightarrow P$. Recall that each preference in the economy $\mathrm{E}$ defines a preference over feasible allocations in $E$.

Definition 25 The economy $\mathrm{E}$ admits a resolution of Arrow's paradox if for any number of voters $j \geq 3$ there exists a social choice function from the space $P=\left\{u_{1}, \ldots, u_{H}\right\}$ of preferences of the economy $\mathrm{E}$ into the space $Q$ of complete transitive preference defined on the space of feasible allocations of $E, \Phi: P^{j} \rightarrow Q$, satisfying Arrow's three axioms. 
Definition 26 A feasible allocation $\left(\alpha_{1}^{k}, \ldots, \alpha_{H}^{k}\right) \in R^{N \times H}$ has utility value $k$, or simply value $k$, if each trader achieves at least a utility level $k$ :

$$
\min _{h \in H}\left\{\left[u_{1}^{k}\left(\alpha_{1}^{k}\right), \ldots, u_{H}\left(\alpha_{H}^{k}\right)\right]\right\}>k .
$$

Definition 27 Arrow's paradox is said to be resolved on choices of large utility value in the economy $\mathrm{E}$ when for all $j \geq 3$ there exists social choice function $\Phi: P^{j} \rightarrow Q$ and $a$ $k>0$ such that $\Phi$ is defined on all profiles of $j$ preferences in $E$, and it satisfies Arrow's three axioms when restricted to allocations of utility value exceeding $k$.

Theorem 28 Limited arbitrage is necessary and sufficient for a resolution of Arrow's paradox on choices of large utility value in the economy $\mathrm{E}$.

Proof. Necessity follows from Proposition 5, since by Arrow's axiom of independence of irrelevant alternatives, the existence of one Condorcet triple of size $k$ suffices to produce Arrow's impossibility theorem on feasible choices of value $k$ in our domain of preferences, see Arrow (1951). Sufficiency is immediate: limited arbitrage eliminates feasible allocation of large utility value by Proposition 4, because it bounds gains from trade. Therefore it resolves Arrow's paradox, because this is automatically resolved in an empty domain of choices.

\subsection{Anonymity and respect of unanimity}

Consider now the second approach to social choice, Chichilnisky [12], [13] which seeks continuous anonymous social choice rules which respect unanimity. The link connecting arbitrage with social choices is still very close but it takes a different form. In this case the connection is between the contractibility of the space of preferences, which is necessary and sufficient for the existence of continuous, anonymous rules which respect unanimity (Chichilnisky and Heal [32]) and limited arbitrage.

Continuity is defined in a standard manner; anonymity means that the social preference does not depend on the order of voting. Respect of unanimity means that if all individuals have identical preferences overall, the social preference is this common preference; it is a very weak version of the Pareto condition. It was shown in Chichilnisky [12] [13] that, for general spaces of preferences, there exist no social choice rules satisfying these three axioms. Subsequently Chichilnisky and Heal [32] established that contractibility is exactly what is needed for the existence of social choice rules.

Formally: consider a general topological space $\chi$ consisting of preferences over the space of allocations $X^{K}$ for $K \geq 2$ individuals. The preferences in $\chi$ need not be those of a market economy. A $K$-profile of individual preferences is a list of preferences for the $K$ individuals, i.e. a $K$-tuple of preferences in the space $\chi$, denoted $\left(\kappa_{1} \ldots \kappa_{K}\right) \in \chi^{K}$. The social choice problem is defined on any space of preferences $\chi$ as the problem of finding for all $K \geq 2$ a map $\phi: \chi^{K} \rightarrow \chi$ such that

(A1) $\phi$ is continuous

$(A 2) \phi$ is symmetric or anonymous, i.e. $\phi\left(\kappa_{1} \ldots \kappa_{K}\right)=\phi\left(\pi\left(\kappa_{1}\right) \ldots \pi\left(\kappa_{K}\right)\right)$ for any permutation $\pi$ of the set $\{1 \ldots K\}$, and

(A3) $\phi$ respects unanimity, i.e. $\phi\left(\kappa_{1} \ldots \kappa_{K}\right)=\kappa$ if $\kappa_{i}=\kappa_{j}=\kappa$ for all $i, j \in\{1 \ldots K\}$.

Axioms $(A 1),(A 2),(A 3)$ were introduced in Chichilnisky [12][13]. A comparison between these axioms and Arrow's classic axioms of social choice is discussed in Section 13. It was proven in Theorem 1 of Chichilnisky [12] that these three axioms are generally 
inconsistent, in the sense that when $\chi$ is the space of all non-trivial smooth preferences defined on Euclidean choice spaces, there exists no map $\phi$ satisfying these three axioms. The result is valid whether or not the preferences admit satiation. However, when respect of unanimity (A3) is replaced by a Pareto condition, the impossibility result of Chichilnisky [12] holds even when the total indifference between all choices is allowed as the social preference. ${ }^{24}$

It is worth observing that the following result is valid for any topology on the space of preferences $T$. In this sense this result is analogous to a fixed point theorem or to a maximization theorem: whatever the topology, a continuous function from a compact convex space to itself has a fixed point and a continuous function of a compact set has a maximum. All these statements, and the one below, apply independently of the topology chosen.

Theorem 29 Let $T$ be a connected space of preferences endowed with any topology. Then $T$ admits a continuous anonymous map $\Phi$ respecting unanimity

$$
\Phi: T^{k} \rightarrow T
$$

for every $k \geq 2$, if and only if $T$ is contractible.

Proof. See Chichilnisky and Heal [32].

The close relation between contractibility and non-empty intersection--which is limited arbitrage-follows from the following theorem:

Theorem 30 Let $\left\{U_{i}\right\}_{i=1 \ldots I}$ be a family of convex sets in $R^{N}$. The family has a nonempty intersection if and oniy if every subfamily has a contractible union:

$$
\bigcap_{i=1}^{I} U_{i} \neq \phi \Leftrightarrow \bigcup_{i \in J} U_{i} \text { is contractible } \forall J \subset\{1 \ldots I\} \text {. }
$$

Proof. See Chichilnisky [15], [19].

This theorem holds for general excisive families of sets, including acyclic families and even simple families which consist of sets which need not be convex, acyclic, open or even connected. This theorem was shown to imply the Knaster Kuratowski Marzukiewicz theorem and Brouwer's fixed point theorem [19], but it is not implied by them.

\subsubsection{Social Allocations}

Consider any space $\chi$ of preferences over the space of allocations $X^{r}$; there are $r \geq 2$ individuals, each with a preference $\kappa_{i} \in \chi, i=1 \ldots r$. The preferences in $\chi$ may or may not be part of a market economy. When a social choice map $\phi: \chi^{r} \rightarrow \chi$ exists satisfying the thre axioms: continuity $(A 1)$, anonymity $(A 2)$, and respect of unanimity $(A 3)$ it defines a social allocation for the space of preferences $\chi$ as follows: For each profile of individual preferences $\left(\kappa_{1} \ldots \kappa_{r}\right) \in \chi^{r}$ a social allocation is a resource allocation in $X^{r}$ which is optimal within the set of feasible allocations $\Upsilon$ according to the social preference $\phi\left(\kappa_{1} \ldots \kappa_{\tau}\right) \in \chi$. Such a resource allocation is located by a social choice rule which satisfies the three ethical axioms $(A 1)(A 2),(A 3)$.

\footnotetext{
${ }^{24}$ The Pareto condition requires that when all preferences in a profile prefer one choice $y$ to another $x$, so does the social preference.
} 


\section{Similarity of preferences}

We aim to define precisely what is meant by preferences being similar, and to show how to define populations of individuals having preferences similar to those in the market $E$. The concepts of similarity and diversity will take the preferences and endowments in the economy $E$ as a benchmark. An essential characteristic of the agents is their global cones. A geometric interpretation of similarity is that a preference $\sigma$ is similar to another preference $\rho$ when $\sigma$ increases along those directions which give $\rho$ unbounded utility gains, and only along those directions. In particular, a preference $\sigma$ is not similar to another $\rho$ if - and only if - there exist a direction along which $\rho$ can achieve unbounded increases in utility, yet along the same direction, $\sigma^{\prime} s$ utility decreases. Intuitively the preferences $\rho$ and $\sigma$ should not be considered similar in this latter case. We shall therefore consider a universe of preferences in which individuals' preferred directions are similar to those of the individuals in the market in the sense that their gradients have positive inner products with the global cones of some trader in the economy $E$.

Formally, consider an economy $E=\left\{X, \Omega_{i}, \rho_{i}, i=1 \ldots H\right\}$. Each preference $\rho_{i}$ in $E$ is defined over $i^{\prime} s$ private consumption set $X$. However, as we saw in Section $2, \rho_{i}$ also defines a preference over all allocations in $X^{r}$ for any number of individuals $r \geq 1$ : an allocation $\left(x_{1} \ldots x_{r}\right) \in X^{r}$ is preferred by individual $i$ in position $j$ to another $\left(y_{1} \ldots y_{r}\right)$ if and only if $\rho_{i}$ prefers $x_{j}$ to $y_{j}$. We may therefore consider $\rho_{i}$ as a preference defined over allocations, using for it the same notation when the meaning is clear from the context.

Consider first the case $X=R^{N}$. Let $\eta$ be any concave, smooth preference defined over allocations in $X^{r}, r \geq 1$. Note that, in general, a preference over allocations may not be monotonic. We seek to formalize the notion that $\eta$ is similar to the preference of trader $i$ in the market $E$ if it agrees with the preference $\rho_{i}$ of $i$ on important choices: the preference $\eta$ must increase in the directions of individual $i^{\prime} s$ global cone. This is formalized in the following definition of similarity to the preference of trader $i$.

Let the normal to the indifference surface of the preference $\eta$ at the allocation $\xi \in X^{r}$ be denoted $G \eta(\xi)$; it is an $N \times r$ vector indicating the direction of increase of preference $\eta$ at the social allocation $\xi \in R^{r}$. This normal always exists and is unique because we assumed that the preference $\eta$ is smooth:

Definition 31 When $X=R^{N}$ an individual preference $\eta$ over resource allocations in $X^{r}$ is said to be similar to the preference of trader $i$ of the economy $E=\left\{X, \rho_{i}, \Omega_{i}, i=1 \ldots H\right\}$ in position $j$ when:

$$
\forall \xi \in X^{r} G \eta^{j}(\xi) \in D_{i}\left(\rho_{i}, \Omega_{i}\right)
$$

where $D_{i}\left(\rho_{i}, \Omega_{i}\right)$ is the market cone of individual $i$. This means that the gradient of $\eta$ has a strictly positive inner product with the vectors of the global cone $A_{i}\left(\rho_{i}, \Omega_{i}\right)$ of individual $i$ in position $j$, as defined in Section 2 . Similarly:

Definition 32 When $X=R_{+}^{N}$, a preference $\eta$ over resource allocations in $X^{r}$ is said to be similar to the preference of trader $i$ of the economy $E=\left\{X, \rho_{i}, \Omega_{i}, i=1 \ldots H\right\}$ in position $j$ when:

$$
\forall \xi \in X^{r} G \eta^{j}(\xi) \in D_{i}^{+}\left(\rho_{i}, \Omega_{i}\right)
$$




\subsection{Private and Public Preferences}

A preference $\eta$ is private when it is indifferent to the consumption of anyone else, and it is public otherwise. Therefore a preference $\rho_{i}$ is private when it is defined over individual $i^{\prime} s$ (private) consumption set $X$; it is public if defined over allocations, $X^{H}$. For any number $r \geq 2$ of individuals, a private preference $\rho_{i}$ defines a public preference: $\left(x_{1} \ldots x_{r}\right) \in X^{r}$ is preferred by individual $i$ in position $j \in\{1 \ldots r\}$ to $\left(y_{1} \ldots y_{r}\right) \in X^{r} \Leftrightarrow x_{j} \succeq_{p_{i}} y_{j}$. When a preference is private and the individual occupies position $j \in\{1 \ldots r\}$, then its normal $G \eta(\xi) \in R^{r \times N}$ has only $N$ non-zero components, thase in position $j$, indicated $G \eta^{j}(\xi)$. A private preference is therefore similar to that of an individual $i$ in the economy $E$ in one position $j$. By contrast, if a preference is not private, it may in principle be similar to those of different individuals $i$ in different positions $j=1 \ldots r$. The results of this paper apply equally well when we consider solely private individual preferences, or when individual preferences are either private or public. When the space of preferences consists solely of private preferences, the concept of respect of unanimity must be modified slightly. ${ }^{25} \mathrm{We}$ consider here spaces consisting of preferences which are either private or public.

We may now define similarity of a preference with respect to a set $K$ of traders in the economy $E$ (as opposed to similarity to the preference of a trader $i$ in position $j \in\{1,2 \ldots, r\}$, which was defined in $(7)$ and $(6))$ :

A private preference $\eta$ over resource allocations in $X^{r}$ is similar to those of a set of traders $K \subset\{1 \ldots H\}$ in the market economy $E$ if:

$$
\exists i \in K \text { and } \exists j \in\{1 \ldots r\} \text { s.t. } \eta \text { is similar to } \rho_{i} \text { in position } j, \forall \xi \in X^{r}
$$

When the preference $\eta$ is public, we have:

$$
\forall j \in\{1 \ldots r\}, \exists i \in K \text { s.t. } \eta \text { is similar to } \rho_{i} \text { in position } j, \forall \xi \in X^{r}
$$

The next step is to define spaces of preferences $P_{E}$ which consist of preferences similar to those of a subset of traders in the market $E$. The intuitive notion is that of a class of preferences $P_{E}$ defined over allocations in $X^{H}$ where each preference in $P_{E}$ is similar to the preference of some trader $i$ within a subset of traders of $E$. Formally, consider the space of allocations for $r \geq 2$ individuals, $X^{r}$, where $X=R^{N}$ or $X=R_{+}^{N}$. Let $j$ denote possible positions, $j=1 \ldots r$ :

Definition 33 A space of preferences $P_{E}$ over allocations $\mathrm{X}^{r}$ is said to contain preferences similar to those preferences of a set of traders $K \subset\{1 \ldots H\}$ in the market economy $E=\left\{X, \rho_{i}, \Omega_{i}, \imath=1 . . H\right\}$, for $X=R^{N}$ or $\mathrm{X}=\mathrm{R}_{+}^{N}$ when

$$
\eta \in P_{E} \Leftrightarrow \eta \text { is a preference similar to those of the set of traders } K \text {. }
$$

The space of preferences $P_{E}$ consists therefore of either private or public preferences over allocations in $\mathrm{X}^{r}$; in either case, its preferences are similar to those of the subset $K$ of traders in the market economy $E$. Note that the preferences in a space $P_{E}$ need not be increasing even when all the preferences in the market are increasing. Similarity only requires that the gradients of preferences in $P_{E}$ be in the dual cones of some preference in the set of traders $K$, and the dual cones of increasing preferences may contain vectors which are not positive.

The following preparatory lemma describes the geometrical structure of spaces of preferences $P_{E}$ which are similar to those in a set $K$ of traders of the economy $E$.

\footnotetext{
${ }^{25}$ Respect of unanimity then means that if all individuals have the same gradient over all possible private choices, then the social preference's gradient over allocations has the same gradient as the traders in each position, for all possible allocations.
} 
Lemma 34 Consider a market economy $E=\left\{X, \rho_{i}, \Omega_{i}, i=1 . . H\right\}$, where $X=R^{N}$ or $X=R_{+}^{N}$. Let $P_{E}$ be a space of preferences over allocations for $r$ individuals $X^{r}$ which are similar to those of a set $K \subset\{1 \ldots H\}$ of traders in $E$. Then when $X=R^{N}$ at each allocation $\xi \in X^{r}$ and for each position $j=1 \ldots r$, the normals to the indifference surfaces of all preferences in $P_{E}$ define the set

$$
N^{K}\left(P_{E}\right)=\left\{\bigcup_{i \in K} D\left(\rho_{i}, \Omega_{i}\right)\right\} \cup\{0\}=N_{K} \cup\{0\} \subset R^{N} .
$$

Furthermore, when every two-trader sub economy of $E$ satisfies limited arbitrage, then $\forall K \subset\{1 \ldots H\}$ the set $N_{K}$ is connected. The set $N_{K}$ is contractible $\forall K \subset\{1 \ldots H\}$ if and only if the market economy $E$ satisfies limited arbitrage. When $X=R_{+}^{N}$ all the above statements hold replacing $N_{K}$ by the set

$$
M_{K}=\left\{\bigcup_{i=1}^{K} \partial D\left(\rho_{i}, \Omega_{i}\right)\right\}
$$

Proof. Since $P_{E}$ consists of preferences over allocations in $X^{r}$ which are similar to those of the subset $K$ of traders in the economy $E$, and these preferences may be private or public, then by definition $(10)(8)(9)(6)$, at each $\xi \in X^{r}$ the gradients in the set $N^{K}\left(P_{E}\right)$ define the set

$$
\left\{\bigcup_{i \in K} D\left(\rho_{i}, \Omega_{i}\right)\right\} \bigcup\{0\} \subset R^{N} .
$$

The next step is to establish that the set $N_{K}$ is connected. The condition of limited arbitrage for any subset of two traders in the statement of this Lemma, is that is that for any two traders $l, j$ in $E$

$$
D\left(\rho_{l}, \Omega_{l}\right) \bigcap D\left(\rho_{j}, \Omega_{j}\right) \neq \emptyset
$$

This implies that the set

$$
N_{K}=\bigcup_{i=1}^{r} D_{i}\left(\rho_{i}, \Omega_{i}\right)
$$

is connected.

Finally we study the contractibility of the set $N_{K}$. Theorem 9 in the Appendix establishes that if $\left\{C_{j}\right\}_{j=1 \ldots J}$ is a family of convex sets and $L \subset J$ then

$$
\begin{gathered}
\bigcup_{j \in L} C_{j} \text { is contractible } \forall L \subset J \\
\Leftrightarrow \forall L \subset J, \bigcap_{j \in L} C_{j} \neq \emptyset .
\end{gathered}
$$

Since the dual cone $\forall j D\left(\rho_{j}, \Omega_{j}\right)$ is a convex set, the second statement in (12) is equivalent to the condition that $E$ has limited arbitrage. The first statement, in turn, states that the set $N_{K}$ is contractible. Therefore Theorem 9 in the Appendix implies that limited arbitrage is satisfied if and only if $\forall K \subset\{1 \ldots H\}$, the set $N_{K}$ is contractible.

The following result links the resolution of the social allocation problem with the resolution of the market allocation problem. A minimal restriction, that every two traders have limited arbitrage, is now imposed in the market economy $E$ to eliminate somewhat pathological economies where no two traders can reach a competitive equilibrium, or those economies where the spaces of preferences similar to those of the traders are discrete or very disconnected:

Assumption (C1): every two-trader sub economy of $E$ has limited arbitrage.

Note that condition $(C 1)$ is not necessary for the equivalence of limited arbitrage and the existence of a competitive equilibrium. The role of this condition is to ensure that the 
set of gradients $N_{K}$ is connected: this was shown in Lemma 2 . Note that $(C 1)$ is rather mild: it certainly does not imply that $N_{K}$ is contractible, nor that the economy $E$ satisfies limited arbitrage, nor that $E$ has a competitive equilibrium. For example, Figure 5 above illustrates an economy where every two dual cones intersect and thus $(C 1)$ is satisfied, but the economy of Figure 5 does not satisfy limited arbitrage, and it does not have a competitive equilibrium.

It seems useful to explain intuitively the role of the limited arbitrage condition in the existence of social choice rules. A condition which is necessary and sufficient for the existence of social choice rules is that the space of preferences should be contractible, Chichilnisky and Heal [32]. Therefore to show the existence of social choice rules on preferences similar to those of the market $E$ we must show that the space of such preferences is contractible. This we do using the condition of limited arbitrage and Theorem 9 in the Appendix.

Limited arbitrage is the non-empty intersection of market cones. However, Theorem 9 proves that the dual cones intersect if and only if their union is contractible. And, by Lemma 1, the union of the market cones is precisely the space where the gradients of the preferences similar to those of the market "live". In other words: the proof that limited arbitrage is necessary for the existence of a social choice rule derives from the results of Lemma 1 above, from Theorem 1 of Chichilnisky and Heal [32], and from Theorem 9 in the Appendix. It may be worth mentioning that preferences which are similar to those in the market $E$ are not necessarily increasing. Furthermore, a space of preferences consisting exclusively of increasing preferences may not admit social choice rules because the union of the market cones may have "holes" and therefore may fail to be contractible.

\subsubsection{Contractibility and Similarity}

Consider a space $P_{E}$ of preferences which are similar to those of the traders in $E$. From Chichilnisky and Heal [32] we know that a social choice rule on this space of preferences exists if and only if the space of preferences $P_{E}$ is contractible, as defined in Section 2 . This condition of contractibility simply means that there exists a continuous way of deforming the preferences through the space $P_{E}$, so that at the end of this process we have complete unanimity. With contractibility of the space $\chi$ we are assured of the existence of a social choice map, and therefore we are assured of a resolution to our resource allocation problem.

But contractibility is a restriction on social diversity, no more and no less. It tests whether there is a way of deforming continuously our space of individual preferences into itself so that at the end of this deformation all the individuals have identical preferences. A discussion of the role of contractibility in limiting social diversity and in public decision making is in Heal [43]. Thus we are back at the source of the problem of resource allocation in markets: individual diversity. As before, we shall focus on the degree of diversity which allows a solution to the resource allocation problem to exist, this time, at the social choice level.

\section{Social Allocations in a Market}

We now specialize the social choice problem to a market economy $E=\left\{X, \rho_{i}, \Omega_{i}, i=\right.$ 1.. $H\}$. Let $P_{E}$ be a space of preferences over resource allocations in $X^{r}, r \geq 2$, consisting of preferences which are similar to those of $K$ traders in the economy $E$ as defined in (10). A social choice map $\phi$ assigns to each profile of $r$ individual preferences in $X^{r}$ another preference in $P_{E}$, the social preference over allocations, $\phi\left(\kappa_{1} \ldots \kappa_{r}\right)=\theta$. Each $\kappa_{j}$ is a preference similar to the preference of an individual $i$ in $E$ and so is $\theta=\phi\left(\kappa_{1} \ldots \kappa_{r}\right)$. 


\subsection{Example: A Classical Social Welfare Function}

Each individual $i=1 \ldots H$ has a utility function $u_{i}: X \rightarrow R$, where $X=R^{N}$ or $X=R_{+}^{N}$. The social preference $W$ over allocations in $X^{H}$ is defined for each allocation $\left(x_{1} \ldots x_{H}\right) \in$ $X^{-H}$ by:

$$
W\left(x_{1} \ldots x_{H}\right)=\sum_{i=1}^{H} u_{i}\left(x_{i}\right) .
$$

Note that the gradient of the function $W: R^{N \times H} \rightarrow R$ at an allocation $\left(x_{1} \ldots x_{H}\right)$ is a vector in $R^{N \times H}$, while each individual utility $u_{i}$ has a gradient in $R^{H}$ at the vector $x_{i}$. In particular. the gradient of the function $W$ is not the sum or any convex combination of the gradients of the individuals $i=1 \ldots H$.

The construction of the social welfare function $W$ of $(13)$ is cardinal, in the sense that it is defined over profiles of individual utilities, and yields a social utility function $W\left(x_{1} \ldots x_{H}\right)$. However, the social welfare function of (13) can be used to define a social choice map in ordinal terms, namely a map from profiles of individual preferences to social preferences. Consider a profile of private preferences $\left(\kappa_{1} \ldots \kappa_{H}\right)$, where $\kappa_{i}$ is a private preference over allocations in $X^{H}$. Then $\forall i \kappa_{i}$ is induced by a utility function over private consumption $u_{i}\left(\kappa_{i}\right): X \rightarrow R$. We may now define the social preference over allocations in $X^{H}$ corresponding to the profile of preferences $\left(\kappa_{1} \ldots \kappa_{H}\right)$, the preference $\Theta\left(\kappa_{1} \ldots \kappa_{H}\right)$, as follows. The normal of $\Theta\left(\kappa_{1} \ldots \kappa_{H}\right)$ at an allocation $\xi=\left(x_{1} \ldots x_{H}\right) \in X^{H}$ is defined as the vector

$$
\begin{gathered}
N \Theta\left(\kappa_{1} \ldots \kappa_{H}\right)(\xi)= \\
\left(\lambda_{1} G_{j} W\left(u_{1}\left(\kappa_{i}\right) \ldots u_{H}\left(\kappa_{H}\right)\right)(\xi), \ldots, \lambda_{H} G_{H} W\left(u_{1}\left(\kappa_{i}\right) \ldots u_{H}\left(\kappa_{H}\right)\right)(\xi)\right),
\end{gathered}
$$

where $G_{j} W$ is the normalized gradient of $W\left(x_{1} \ldots x_{H}\right)$ with respect to $x_{j}$, i.e.

$$
G_{j} W(\xi)=\left(\partial W / \partial x_{j}\right)(\xi) \in R^{N},
$$

and $\forall j, \lambda_{j} \in R_{+}$. The role of the real numbers $\lambda_{j}$ in (14) is to normalize the right hand side of the expression (14) so that it defines a vector of length one as it corresponds to the normal of a smooth preference defined in Section 2 (Debreu [34]). Expressions (13) and (14) define a map from profiles of private preferences over allocations in $X^{H}$, into preferences over allocations in $X^{H}$ :

$$
\left(\kappa_{1} \ldots \kappa_{H}\right) \rightarrow \Theta\left(\kappa_{1} \ldots \kappa_{H}\right)
$$

Clearly, the definition in (15) is the ordinal version of the cardinal construction in (13).

The interest of the social welfare function $\theta$ defined in (15) is that it provides an example of the type of structure for social choice with which we work in this paper. The similarities are: $\theta$ is defined over profiles of private preferences over allocations, and it assigns to such a profile another preference over allocations which is typically a public preference. If the preferences in the profile $\left(\kappa_{1} \ldots \kappa_{H}\right)$ are those of a market economy $E=\left\{X, \kappa_{i}, \Omega_{i}, i=1 \ldots H\right\}$, then the social preference assigned to this profile $\Theta\left(\kappa_{1} \ldots \kappa_{H}\right)$ is similar to those of the traders in $E$ as defined in (10) above. Furthermore, the map $\Theta$ is continuous on its arguments and it respects unanimity. Therefore the map $\Theta$ satisfies many of the properties required of our social choice functions.

The differences with our framework are as follows: firstly, $\Theta$ is defined solely on private preferences, while we allow social choice maps which are more general, defined over both private and public preferences over allocations. Secondly, the map $\Theta$, by its construction, is not anonymous. Indeed, the map $\Theta$ assigns individual $i$ a dictatorial power over the $i$ th position in the allocation. 


\subsection{Example: The Convex Addition of Gradients}

As pointed out in 5.1, the gradient of the classical welfare function $W$ defined in (13), (14) is neither the sum nor the convex combination of the individual utilities' gradients. A natural question is whether a construction based on the sum or a convex combination of the individual utilities's gradients could yield an adequate social preference. Such construction would have the advantage that it is defined generally, without reference to additional conditions such as limited arbitrage. The following example shows that such a construction, although appearing to be natural, typically does not work. Indeed we argue below that it only works properly when the limited arbitrage condition is satisfied.

Consider for example an economy with two agents each with a linear utility defined on the consumption set $X=R^{2}$. The two utilities are different. The global cones $A_{1}, A_{2}$ of the two linear preferences are two different half spaces, and the corresponding market cones $D_{1}, D_{2}$ are the two gradient vectors indicated in Figure 9.

\section{Figure 9}

Since the two vectors are different, the market cones do not intersect, and limited arbitrage is violated. Now consider the linear preference $\rho$ over allocations in $R^{2} \times R^{2}$ with gradient $\left(D_{3}, D_{3}\right)$, where $D_{3}$ is a convex combination of $D_{1}$ and $D_{2}$ as shown in Figure $10, D_{3}=\lambda D_{1}+(1-\lambda) D_{2}, 0<\lambda<1$.

\section{Figure 10}

A problem arises in that although the initial endowment allocation $\left(\Omega_{1}, \Omega_{2}\right)$ is strictly preferred by both individuals to the allocation denoted $\left(\theta_{1}, \theta_{2}\right)$ in Figure 10 , the social preference $\rho$ strictly prefers $\left(\theta_{1}, \theta_{2}\right)$ to $\left(\Omega_{1}, \Omega_{2}\right)$ instead.

This property of $\rho$ contradicts the standard Pareto condition which requires that when everyone strictly prefers a given allocation to another, so should the social preference. The reason for this contradiction is that the two dual cones do not intersect. When the dual cones intersect, then a vector in this intersection defines the gradient of a linear preference which respects the Pareto condition: this follows directly from the definition of dual cones. Otherwise, no preference exists respecting the Pareto condition.

Another way of looking at the same problem is that the social preference defined by the addition of the two gradients is not similar to the preferences with gradients $D_{1}$ and $D_{2}$, as defined in Section 9. Therefore the addition of gradients cannot define a map from profiles of similar preferences into similar preferences as required. This example shows that the convex sum of gradients cannot be used generally as a way of defining appropriate social choice rules: the preference with gradients $D_{3}=\lambda D_{1}+(1-\lambda) D_{2}, 0<\lambda<1$, is not an adequate social preference for the profile $\left(D_{1}, D_{2}\right)$.

The only possibility of respecting the similarity of preferences or the Pareto condition, would be to assign the social preference with gradient equal to either $D_{1}$ or to $D_{2}$ to the profile $\left(D_{1}, D_{2}\right)$. This would respect the similarly condition, and would define a continuous, anonymous social choice map respecting unanimity on the space of preferences $P_{E}=$ $\left\{D_{1}, D_{2}\right\}$. But this is possible only because the space $P_{E}$ is a discrete case consisting only of two elements, the two linear preferences. In the general case in which the space $P_{E}$ is connected, a continuous anonymous social choice rule satisfying unanimity exists if and only if $P_{E}$ is contractible, as proved in Chichilnisky and Heal [32]. And, as shown in the Appendix Theorem 9 and Corollary 2 , limited arbitrage is actually equivalent to the contractibility of the spaces of preferences.

Our next step is to extend and refine the classical welfare function $W$ defined in (13). The welfare function $W$ is a cardinal construct, while we wish to provide an ordinal one: a social choice map defined over profiles of private and public preferences over allocations, 
Figure ?

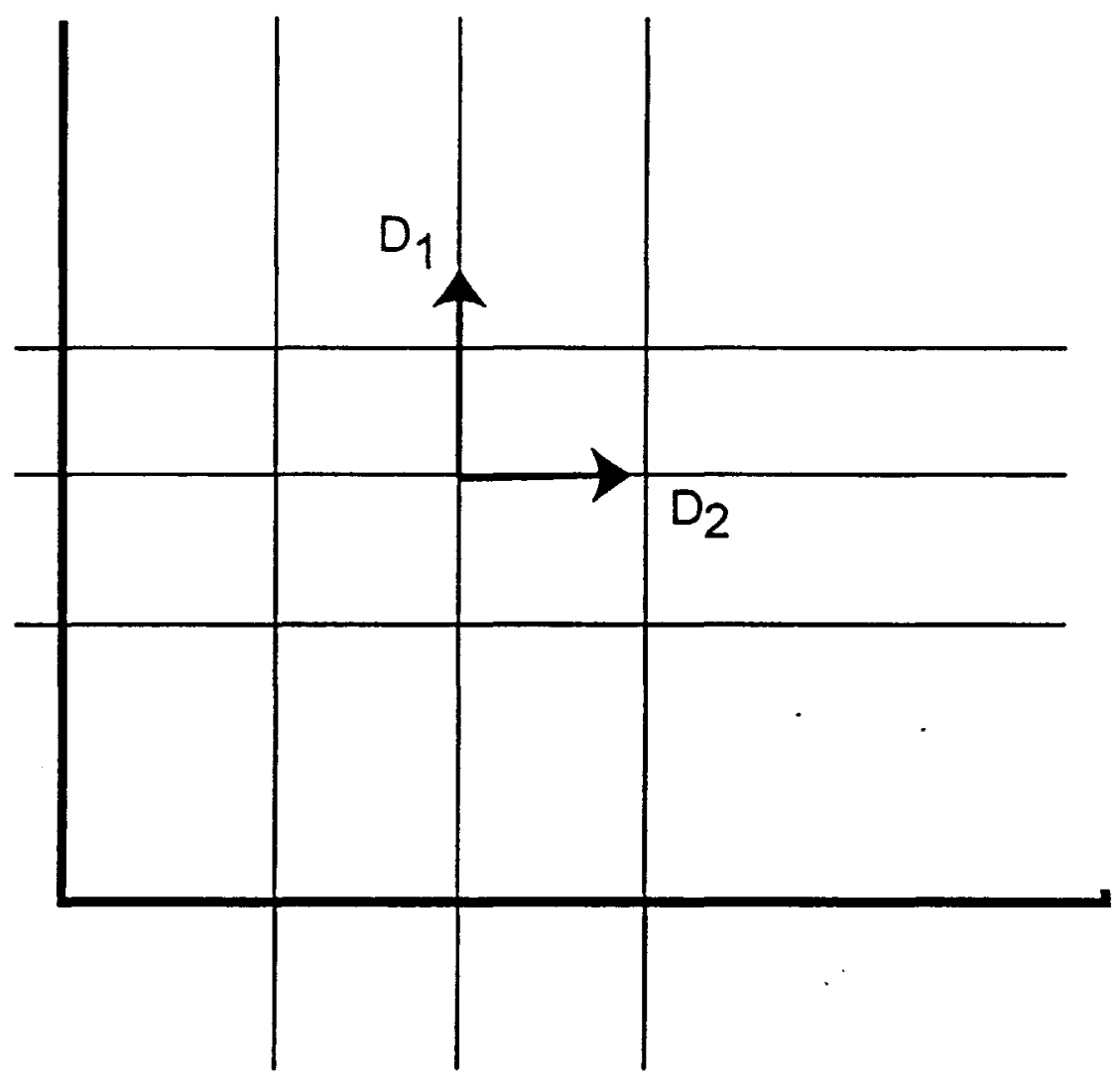




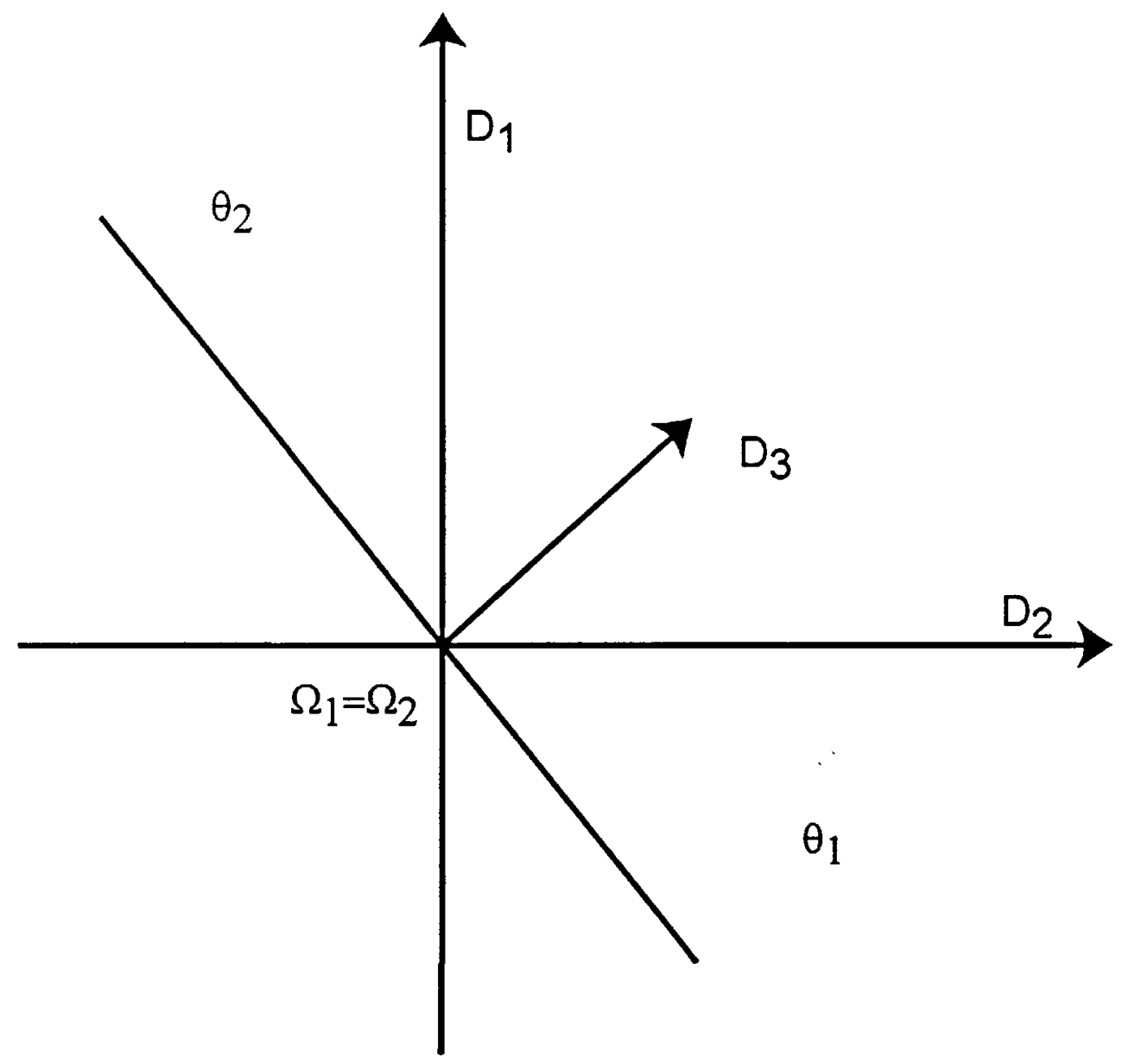

Figure 1D 
preferences which are similar to those of a market economy $E$. We seek an anonymous social choice map, a requirement that neither $W$ in (13) nor its ordinal counterpart $\Theta$ in (15), satisfy. Theorem 7 in Section 11 establishes that this task can be accomplished when the preferences in the market economy $E$ satisfy limited arbitrage, and therefore by Theorem 9 and Corollary 2 of the Appendix, when the market $E$ has a competitive equilibrium. and only then.

Assume now that a social choice map 0 with the desired properties $(A 1)(A 2)(A 3)$ exists. The existence of a social choice map $\phi$ solves the resource allocation problem from the point of view of social choice:

We say that the social allocation problem is resolved for preferences similar to those of the market $E=\left\{X, \rho_{i}, \Omega_{i}, i=1 . . H\right\}$ when for any space of preferences $P_{E}$ over allocations in $X^{r}$ similar to a set of traders $K \subset\{1 \ldots H\}$ in $E$ there exists a continuous anonymous social choice map $\phi:\left(P_{E}\right)^{r} \rightarrow P_{E}$ respecting unanimity.

Consider a market economy $E=\left\{X, \rho_{i}, \Omega_{i}, i=1 . . H\right\}$ having a social choice map $\omega:\left(P_{E}\right)^{H} \rightarrow P_{E}$ satisfying the axioms $(A 1)(A 2)(A 3)$ on the space of preferences $P_{E}$ similar to those of the economy $E$.

A social allocation for $E$ is a feasible allocation of $E$ which is optimal in the space of feasible allocations $\Upsilon=\left\{x_{1} \ldots x_{H}: \sum x_{i}=\Omega\right\}$ according to the social preference $\phi\left(\rho_{1} \ldots \rho_{H}\right)$.

\section{Comparing the two resource allocations problems}

\subsection{Existence of market allocations}

As seen in Section 3 above, even when all individual preferences are smooth, concave and increasing, and when the consumption sets are positive orthants, not all Arrow-Debreu exchange economies have a competitive equilibrium. The problem is quite general and it occurs in economies with any number of individuals and of goods. ${ }^{26}$ Limited arbitrage limits precisely the degree of diversity among the agents of the economy to one at which market equilibrium will exist. Indeed, for economies with consumption bounded below, $X=R_{+}^{N}$, such limits on diversity are implicit in Arrow's resource relatedness [3] and in McKenzie's irreducibility condition [47][48][49]. All these conditions ensure that the endowments of any household are desired, directly or indirectly, by others, so that their incomes cannot fall to zero. In this case our limited arbitrage condition is always satisfied. Yet zero income by itself does not rule out the existence of a competitive equilibrium; there may be economies with a competitive equilibrium in which some consumers have zero income. Figure 7 above illustrates. Furthermore an allocation where some individuals have zero income reflects a real problem: the fact that some individuals are considered

\footnotetext{
${ }^{26}$ Other concepts of market equilibrium could be utilized to define a market allocation, such as for example the quasi-equilibrium or pseudoequilibrium introduced by Debreu [37], or as defined in Arrow and Hahn [3]. These are closely related definitions; they define allocations where individuals minimize cost rather than maximizing utility. When individuals' incomes are strictly positive, these concepts agree with the competitive market equilibrium (Arrow and Hahn [3], Chapter 4) but as Arrow and Hahn point out, the condition that all individuals should have strictly positive endowments of all goods is unrealistic ([3], Chapter 4, p. 80. Quasi-equilibrium allocations have the advantage that they always exist with continuous concave preferences and with positive orthants as consumption sets. However, the competitive equilibrium stands alone in terms of its welfare properties: quasi-equilibrium equilibrium allocations are not generally Pareto efficient. Therefore the main justification for using market allocations, which is efficiency, is lost. For this reason we concentrate here on competitive equilibrium allocations. For a study of limited arbitrage and quasiequilibrium, see Chichilnisky [26].
} 
worthless, that they have nothing to offer that others want. Our condition of limited arbitrage brings out the issue of diversity by focusing on the problem of zero income individuals. It does not attempt to rule out the case of individuals with zero income; instead, it seeks to determine if society's evaluation of their worthlessness is widely shared.

\subsection{Existence of Social Allocations}

Within the same type of market economy studied above $E=\left\{X, \Omega_{i}, \rho_{i}, i=1 \ldots H\right\}$, the social allocation problem can be defined as follows. Find a social choice rule $\phi$ satisfying the required axioms $(A 1)(A 2)(A 3): \phi$ must be defined on a space of preferences which contains those of the market $E$ and is otherwise large enough to satisfy Kant's universality criterion. The problem has generally no solution because a social choice rule satisfying the required axioms may not exist, Chichilnisky [12]. Therefore a social contract promising a solution which satisfies the ethical principles agreed and having a completely universal domain of preferences, may not deliver. Again, conditions are needed. Here, as in the case of the market equilibrium, the conditions should be on the exogenous data which identify the economy $E$, namely on the individuals' endowments $\Omega_{i}$ and their preferences $\rho_{i}$.

We know that the space of all smooth preferences over allocations in $X^{H}$ is too large: the three axioms of continuity, anonymity and respect of unanimity are inconsistent in that case and no social choice rule satisfying them exists, Chichilnisky [12]. We must therefore search for a universe of preferences and endowments in which a social choice rule $\phi$ does exist. Such a universe must contain the preferences of our economy $E$, and we shall require that it should consist of individuals who share the essential characteristics of the economy $E$. The motivation is to compare the types of restrictions needed for existence of a competitive equilibrium with those required for the existence of a social choice map.

\section{Linking Markets, Arbitrage and Social Choice}

This section provides the main results linking the two forms of resource allocation: by markets and by social choices.

Theorem 35 Consider an economy $E=\left\{X, \rho_{i}, \Omega_{i}, i=1 \ldots H\right\}, H \geq 2, X=R^{N}$ or $X=R_{+}^{N}, N \geq 1$ satisfying $(C 1)$.

The following properties are equivalent

(a) The market economy $E$ has a competitive equilibrium

(b) Every sub economy of $E$ with at most $N+1$ traders has a competitive equilibrium

(c) E has limited arbitrage

(d) $E$ has limited arbitrage for any subset of individuals with no more than $N+1$ members.

(e) For any space of preferences $P_{E}$ similar to those of a subset $K$ of market traders, there exists a continuous anonymous social choice map $\phi:\left(P_{E}\right)^{r} \rightarrow P_{E}$ respecting unanimity, for all $r \leq N+1$, and

(f) The economy $E$ has a nonempty core.

Proof. The proof that $(a) \Leftrightarrow(c)$ is in [26]. Next I establish the equivalence between (a) and $(\mathrm{e})$.

Case 1: the consumption set $X=R^{N}$. We first show that limited arbitrage is a necessary condition for the existence of the social choice map $\phi$ for all $K \subset\{1 \ldots H\}$ and all $k \geq 2$ satisfying the three axioms $(A 1)(A 2)(A 3)$.

Assume that such a social choice map $\phi$ exists for all $r \geq 2$. Let $L P_{E}$ be the subspace of $P_{E}$ consisting of all its linear preferences, i.e. those preferences within the space $P_{E}$ 
which are representable by linear utility functions having their gradients, in each position $j=1 \ldots r$, contained in the set $N_{K} \cup\{0\} \subset R^{N}$. Let in $: L P_{E} \rightarrow P_{E}$ denote the inclusion map. Note that each preference $\nu \in L P_{E}$ is uniquely identified by the normal $N \nu \in R^{N r}$ to one of its indifference surfaces: by linearity all such normals are the same. Therefore

$$
L P_{E} \approx\left(N_{K} \cup\{0\}\right)^{r},
$$

i.e. the space of linear preferences $L P_{E}$ is homeomorphic to the product space $\left(N_{K} \cup\{0\}\right)^{r}$. Now consider an allocation $\xi \in X^{K}$ and define the map $\pi: P_{E} \rightarrow L P_{E}$ so that

$$
\pi(\kappa)=\kappa \xi
$$

where $\kappa \xi \in L P_{E}$ is the linear preference over allocations in $X^{r}$ having as its gradient in $R^{N r}$ the vector $G \kappa(\xi)$ which is the normal to the indifference surface of the preference $\kappa$ at $\xi$. Both maps in and $\pi$ are continuous in their domains.

By assumption, there exists a social choice map $\phi:\left(P_{E}\right)^{r} \rightarrow P_{E}$ for any $r \geq 2$, satisfying the three axioms. Now consider for any $r \geq 2$ the map induced by the composition of $i n$ and $\phi$, defined by:

$$
\begin{gathered}
\psi:\left(L P_{E}\right)^{r} \rightarrow L P_{E}, \\
\psi\left(\nu_{1} \ldots \nu_{r}\right)=\pi\left(\phi\left(i n\left[\nu_{1}\right] \ldots i n\left[\nu_{r}\right]\right)\right) .
\end{gathered}
$$

The map $\psi:\left(L P_{E}\right)^{r} \rightarrow L P_{E}$, is continuous, anonymous and respects unanimity by construction, because $\phi$ satisfies these three properties. Since by $(16) \forall j, L P_{E} \approx\left(N_{K} \cup\right.$ $\{0\})^{r}, \psi$ defines a map $\Lambda:\left[\left(N_{K} \cup\{0\}\right)^{r}\right]^{r} \rightarrow\left(N_{K} \cup\{0\}\right)^{r}$ for all $r \geq 2$ satisfying the three axioms $(A 1)(A 2)(A 3)$. Since $\Lambda$ is continuous and respects unanimity, it maps each connected component of the space $\left(N_{K} \cup\{0\}\right)^{r}$ into itself. In particular, the restriction of the map $\Lambda$ to the connected component $\left[\left(N_{K}\right)^{r}\right]^{r}$ of $\left[\left(N_{K} \cup\{0\}\right)^{r}\right]^{r}$, denoted $\Lambda /\left(N_{K}\right)^{r}$, maps $\left[\left(N_{K}\right)^{r}\right]^{r}$ into $\left(N_{K}\right)^{r}$, i.e. $\Lambda /\left(N_{K}\right)^{r}:\left[\left(N_{K}\right)^{r}\right]^{r} \rightarrow\left(N_{K}\right)^{r}$, and it satisfies the three axioms $(A 1)(A 2)(A 3)$. By Theorem 1 of Chichilnisky and Heal [32] such a map $\Lambda$ exists if and only if the space $\left(N_{K}\right)^{r}$ is contractible for all $r \geq 2$; this in turn is true if and only if the space $N_{K}$ is contractible - see Section 2. Therefore the contractibility of the space $N_{K}$ is necessary for the existence of the social choice map $\phi$. But Theorem 9 proves that $N_{K}$ is contractible for all $K \subset\{1 \ldots H\}$ if and only if the limited arbitrage condition is satisfied. This completes the proof of necessity of limited arbitrage.

We now turn to the proof of sufficiency of limited arbitrage. By definition, limited arbitrage implies the existence of a non-zero vector $v \in R^{N}$ in the intersection of all the market cones:

$$
v \in \bigcap_{i=1}^{H} D\left(\rho_{i}, \Omega_{i}\right) .
$$

Furthermore, by definition of the space $P_{E}$, all the indifference surfaces of any preference in $P_{E}$ must intersect the ray defined by the vector $(v \ldots v)$ in the space of allocations $X^{N r}$. Therefore the conditions required in Chichilnisky [11] fthe existence of a social choice map $\phi:\left(P_{E}\right)^{r} \rightarrow P_{E}$ satisfying the thre axioms $(A 1)(A 2)(A 3)$ are satisfied for the space $P_{E}$. This completes the proof of sufficiency of limited arbitrage.

Case 2 . Here $X=R_{+}^{N}$. The proof in this case is the same replacing the set of gradients $N_{K}$ by the set

$$
M_{K}=\bigcup_{i=1}^{K} \partial D\left(\rho_{i}, \Omega_{i}\right) .
$$

Finally, the proof that $(a) \Leftrightarrow(b)$ and $(b) \Leftrightarrow(d)$ follows directly from Theorem 9 the Appendix. That $(c) \Leftrightarrow(d)$ follows from [26] and Theorem 9 in the Appendix. The proof that $(c) \Leftrightarrow(e)$ follows from Theorem 3 . 
Theorem 36 Consider a market economy $E=\left\{X, \rho_{i}, \Omega_{i}, i=1 \ldots H\right\}$. Any market allocation $\left(x_{1}^{*} \ldots x_{H}^{*}\right) \in \operatorname{Int}\left(X^{H}\right)$ of $E$ is also a social allocation for $E$.

Proof. When a market allocation for $E$ exists, $E$ has a competitive equilibrium described by a price vector $p^{*}$ and an allocation $\left(x_{1}^{*} \ldots x_{H}^{*}\right) \in X^{H}$ which is individually optimal within the budget sets and which clears all the markets. We shall show that if this market allocation $\left(x_{1}^{*} \ldots x_{H}^{*}\right)$ is interior to $X^{H}$, then it is also a social allocation. This means that any space of preferences $P_{E}$ defined over allocations for the $H$ traders, namely on $X^{H}$, and consisting of preferences which are similar to those of the $\mathrm{H}$ traders in the economy $E=\left\{X, \rho_{i}, \Omega_{i}, i=1 \ldots H\right\}$, admits a social choice map $\Psi:\left(P_{E}\right)^{H} \rightarrow P_{E}$ satisfying the axioms $(A 1)(A 2)(A 3)$, and such that $\left(x_{1}^{*} \ldots x_{H}^{*}\right)$ optimizes the social preference $\Psi\left(\rho_{1} \ldots \rho_{H}\right)$ over the set of all feasible allocations $\Upsilon$ of the economy $E$.

Since a market equilibrium exists, by Theorem 7 there exists a social choice map $\phi$ : $\left(P_{E}\right)^{H} \rightarrow P_{E}$ satisfying the three axioms $(A 1)(A 2)$ and $(A 3)$, for the space of preferences $P_{E}$ similar to the $H$ traders in the economy $E$. By Theorem 3 we also know that limited arbitrage is satisfied, i.e. $\bigcap_{i=1}^{H} D\left(\rho_{i}, \Omega_{i}\right) \neq \emptyset$, since by assumption $E$ has a competitive equilibrium.

We now use a partition of unity on the space $P_{E}$ as defined in Section 2 in order to define a modification of the social choice map $\phi$, called $\Psi$, which also satisfies the three axioms, and according to which the equilibrium allocation is optimal within all feasible allocations for the social preference $\Psi\left(\rho_{1} \ldots \rho_{H}\right)$. Note that by the results of Chichilnisky [11] the space $P_{E}$ is contained in a manifold which is the inverse image under a smooth retraction of a linear space, the space spanned by the vector $(v \ldots v) \in R^{N \times H}$, where $v \in \bigcap_{i=1}^{H} D\left(\rho_{i}, \Omega_{i}\right)$. Therefore the space $P_{E}$ is Hausdorff and we can therefore apply a partition of unity, see the Appendix.

Let $U$ be the set of preference profiles in $P_{E}$ consisting of the profile $\left(\rho_{1} \ldots \rho_{H}\right)$ and of all its permutations,

$$
\left.U=\bigcup \pi\left\{\left(\rho_{\pi_{1} \ldots \rho_{\pi_{H}}}\right)\right\}, \text { for all permutations } \pi \text { of the set }\{1 \ldots H\}\right\} .
$$

The set $U$ consists of finitely many points in $\left(P_{E}\right)^{H} ; U$ is disjoint from the diagonal $\Delta\left(P^{E}\right)^{H}=\left\{\left(\kappa_{1} \ldots \kappa_{H}\right) \in\left(P^{E}\right)^{H}: \forall i, j \kappa_{i}=\kappa_{j}\right\}$, because the profiles in $U$ consist of private preferences each defined over a different position. Since $\left(P_{E}\right)^{H}$ is contained in a manifold, it is a Hausdorff space.

We shall now construct a new social choice map $\Psi$ with the desired properties by using a partition of unity for $\left(P_{E}\right)^{H}$, as defined in Section 2. Using this partition of unity we modify the map $\phi:\left(P_{E}\right)^{H} \rightarrow P_{E}$ to obtain another continuous anonymous map $\psi:\left(P_{E}\right)^{H} \rightarrow P_{E}$, which differs from $\phi$ only in an open neighborhood $\theta(U)$ of the set $U$, and is otherwise identical to $\phi$. Within the set $U$ the new map satisfies:

$$
\Psi\left(\rho_{1} \ldots \rho_{H}\right)=\Psi\left(\rho_{\pi_{1}} \ldots \rho_{\pi_{H}}\right)=\kappa_{p^{*}}
$$

for all permutations $\pi$ of the set of indices $\{1 \ldots H\}$, where in $(17) \kappa_{p^{*}}$ is the linear preference over allocations in $X^{H}$ with gradient vector $\left(\lambda_{1} D u_{1}\left(x_{1}^{*}\right) \ldots \lambda_{H} D u_{H}\left(x_{H}^{*}\right)\right)$, for some vector $\left(\lambda_{1} \ldots \lambda_{H}\right) \in R_{+}^{H}, D u_{i}\left(x_{i}^{*}\right)$ is the gradient of $u_{i}$ at $\left(x_{i}^{*}\right)$ for a utility $u_{i}$ which represents $\rho_{i}$, and where

$$
\Psi\left(\kappa_{1} \ldots \kappa_{H}\right)=\phi\left(\kappa_{1} \ldots \kappa_{H}\right)
$$

if $\left(\kappa_{1} \ldots \kappa_{H}\right) \notin \theta(U)$. The map

$$
\Psi:\left(P_{E}\right)^{H} \rightarrow P_{E}
$$

satisfies the three axioms by construction. We shall now show that the competitive equilibrium allocation $\left(x_{1}^{*} \ldots x_{H}^{*}\right) \in X^{H}$ is a social allocation for the economy $E$ with the social 
choice map $\Psi$. Since the allocation $\left(x_{1}^{*} \ldots x_{H}^{*}\right) \in \operatorname{Int}\left(X^{H}\right)$ by the assumptions of the Theorem and is the equilibrium allocation corresponding to the price vector $p^{*}$, there exists a vector $\left(\lambda_{1}^{*} \ldots \lambda_{H}^{*}\right) \in R_{+}^{H}$, such that:

$$
\begin{gathered}
W\left(x_{1}^{*} \ldots x_{H}^{*}\right)=\operatorname{Max}_{\left(x_{1} \ldots x_{H}\right) \in \Upsilon} W\left(x_{1} \ldots x_{H}\right) \\
\text { where } W\left(x_{1} \ldots x_{H}\right)=\sum_{i=1}^{H} \lambda_{i}^{*} u_{i}\left(x_{i}\right),
\end{gathered}
$$

and where the utility $u_{i}: X \rightarrow R$ represents the preference $\rho_{i}$. Now choose the vector $\left(\lambda_{1} \ldots \lambda_{H}\right)$ in the definition of $\Psi$ following (17)to be the vector $\left(\lambda_{1}^{*} \ldots \lambda_{H}^{*}\right)$ in (18). Then. with this definition of $\left(\lambda_{1} \ldots \lambda_{H}\right)$, the allocation $\left(x_{1}^{*} \ldots x_{H}^{*}\right)$ maximizes the social preference $\Psi\left(\rho_{1} \ldots \rho_{H}\right)$ over the feasible set $\Upsilon$. The allocation $\left(x_{1}^{*} \ldots x_{H}^{*}\right) \in X^{H}$ is therefore a social allocation for the social choice map $\Psi$ and the economy $E$, as we wished to prove.

\section{Social Diversity and a Topological Invariant for Markets}

As defined above, the economy is socially diverse when $\bigcap_{h=1}^{H} D_{h}=\phi$, see also Chichilnisky [26]. Diversity admits different "shades", which can be measured by the smallest number of market cones which do not intersect. I showed in [26] that the resource allocation properties of the economy $E$ can be described simply in terms of a topological invariant, which is given by a family of cohomology rings denoted $C H(\mathrm{E})$.

\section{Related Literature}

\subsection{Comparison with Arrow's axioms}

It seems worth comparing our axioms with Arrow's [2] classic axioms of social choice. Arrow's axioms are more suitable for finite set of choices, such as voting among a finite set of $n$ candidates. Instead, we are choosing here among an infinite set of choices, namely among the set $\Upsilon$ of all feasible allocations in Euclidean space.

The three axioms $(A 1)(A 2)(A 3)$ are different, indeed not comparable, to Arrow's [2]. The anonymity condition $(A 2)$ is stronger than Arrow's non-dictatorship axiom, because the former requires equal treatment while the latter eliminates only extreme inequality of treatment. Respect of unanimity $(A 3)$ is strictly weaker than his Pareto condition, since respect of unanimity is only binding when all preferences within a profile are identical. Instead, the Pareto condition applies to any profile of preferences which, equal or not, prefer a given choice $x$ to another $y$. Finally, Arrow does not consider continuity $(A 1)$ as we do, and we do not require Arrow's axiom of independence from irrelevant alternatives, an axiom which has been somewhat controversial. In other words: neither set of axioms implies the other.

Continuity is required here on the grounds of statistical tractability: it implies that the sampling of populations' preferences will approach the true distribution provided the grid of observations in the sample is fine enough. The continuity axiom makes this formulation of social choice better suited to continuous sets of choices and to connected sets of preferences. This is because when the space of preferences is discrete or finite-as it would be when there are finitely many choices--then the space of preferences is itself finite and therefore continuity is a vacuous requirement. Recent work by Baigent [5][6] and by Nitzan [52] has extended the axiom (A1) of continuity to one of "proximity" 
of preferences, a concept which is appropriate for discrete spaces of preferences and for preferences over finitely many choices. Using "proximity" instead of "continuity", and preserving the other two axioms-anonymity and respect of unanimity-Baigent [5][6] proved the impossibility results of Chichilnisky [12] for the case of finitely many choices. Chichilnisky [14] has recently shown the connection between the existence of social choice rules and the manipulation of non cooperative games.

What makes the axioms used in this paper particularly well suited for our problem is that they lend themselves naturally to the study of preferences and choices similar to those which are studied in market economics. Furthermore, with these axioms there exist simple necessary and sufficient conditions for resolving the social choice paradox, Chichilnisky and Heal [32]; this is not true for Arrow's axioms.

Recent results by Y. Baryshnikov [7] highlight a topological connexion between Arrow's impassibility theorem and my own. This is a somewhat surprising result: using algebraic topology, it shows that the condition of independence of irrelevant alternatives can be used to prove that the 'nerve' of a family of sets naturally associated with all preferences over a finite set of choices, has the topological structure of a sphere. Therefore the non-existence theorem in [12], which applies to spheres, implies Arrow's imposssibility theorem in this context.

\section{Conclusions}

On the basis of previous results [18], [21], [22], and [23], I established that limited arbitrage is a necessary and sufficient condition for the existence of social choice maps on spaces of preferences similar to those of a market economy. The same condition-limited arbitrageis necessary and sufficient for the existence of a competitive equilibrium and the core in a market economy [16], [31], [24], [26], [21]. Furthermore, I showed that a market allocation is always a social allocation. In this sense, the results of this paper unify three forms of resource allocation-by markets, games and by social choice - which have developed and remained separate until now.

I have chosen competitive equilibrium allocations-rather than other concepts of market equilibrium - because of the Pareto efficiency of competitive equilibrium. This property is generally lost in weaker forms of market equilibrium, such as quasi-equilibrium and compensated equilibrium. I considered economies with or without bounds on short sales.

I have concentrated on three axioms of social choice: continuity, anonymity and respect of unanimity, introduced in [12], which are particularly well suited for problems where the choices are elements in Euclidean space, and for which necessary and sufficient conditions exist for resolving the social choice problem [32]. Arrow's [2] classic axioms appear to be better suited for problems with finitely many choices. As already pointed out, however, it is possible to draw a close topological link between Arrow's theorem and the impossibility theorem in [12]. The choice of axioms in the latter has led to necessary and sufficient "domain" restrictions for a resolution of the social choice paradox, proven in Chichilnisky and Heal [32]. In addition, the condition of limited arbitrage characterizes those economies where the traders do not have Condorcet triples (i.e. cyclical behavior) of every utility value, and those where Arrow's impossibility theorem can be resolved.

The crucial condition in all this is the contractibility of the space of preferences, which is a measure of social diversity [43]. Limited arbitrage is closely connected with contractibility. A key element in the results is the necessary and sufficient conditions for the non-empty intersection of a family of sets in Theorem 5, Chichilnisky [15],[19]: these require the contractibility of the union of all subfamilies of these sets. Limited arbitrage is defined as the non-empty intersection of the market cones of the traders, 
which we now know to be equivalent to a contractibility condition. Thus I have linked limited arbitrage, which is needed for the existence of a market equilibrium and the core, with the contractibility of the space of preferences, which is needed for a resolution of the social choice problem. The same restriction on social diversity is therefore necessary and sufficient for the successful resolution of all three problems of resource allocation.

\section{Appendix}

0. Partitions of Unity. Assume that $Y$ is a Hausdorff space [46]. If $U, V$ are two open neighborhoods of $x \in Y, U \subset V, U \neq V$, a continuous map $v: Y \rightarrow R$ is called a partition of unity for $Y, U, V$, if: $\forall y \in Y-V, v(y)=0, \forall y \in U, v(y)=1$, and $\forall y \in Y, 1 \geq v(y) \geq 0$.

Such partitions of unity always exist in Hausdorff spaces, [46]. A partition of unity is used to construct new maps from old ones, having specific values. For any $f: Y \rightarrow Z$ any $y \in Y, z \in Z$, and any open neighborhood $U$ of $y \in Y$, there exists a continuous map $g: Y \rightarrow Z$ such that $g(y)=z$ and $g(x)=f(x) \forall x \in X-U$. If the map $f$ is symmetric, $g$ can be constructed to be symmetric also.

\section{Intersection and contractibility}

Theorem 37 Consider a family $\left\{U_{i}\right\}_{i=1 . . n n}$ of convex sets in $R^{m} n, m \geq 1$. Then

$$
\bigcap_{i=1}^{n} U_{i} \neq \emptyset \text { if and only if } \bigcap_{j \in J} U_{i} \neq \emptyset
$$

for any subset of indices $J \subset\{1 \ldots n\}$ having at most $m+1$ elements. For a proof see Chichilnisky [19].

Corollary 38 A market economy $E$ as defined in Section 2 with $n$ traders and $m$ commodities satisfies limited arbitrage if and only if every sub economy of $E$ with at most $m+1$ traders satisfies limited arbitrage.

For a proof see Chichilnisky [19].

\section{References}

[1] Arrow, K. J. (1951) "An Extension of Basic Theorems of Classical Welfare Economic$\mathrm{s"}$ in J. Neyman (ed.) Proceedings of the Second Berkeley Symposium on Mathematical Statistics and Probability, Berkeley: University of California Press, p. 507-532.

[2] Arrow, K. (1953) Social Choice and Individual Values, Cowles Foundation Monograph, John Wiley.

[3] Arrow, K. and F. Hahn (1971) General Competitive Analysis, North Holland, 1986.

[4] Arrow, K. and G. Debreu (1954) "Existence of an equilibrium for a competitive economy" Econometrica 22, 264-90.

[5] Baigent, N. (1984) "A Reformulation of Chichilnisky's Theorem", Economic Letters, 23-25.

[6] Baigent, N. (1987) "Preference Proximity and Anonymous Social Choice" Quarterly Journal of Economics, 162-194. 
[7] Baryshnikov, Y. (1993) "Unifying Impossibility Theorms: A Topological Approach" Advances in Applied Mathematics, 14, 404-415.

[8] Black, D. (1948) "On the Rationale of Group Decision Making" Journal of Political Economy, 56.

[9] Black, D. (1948a) "The Decisions of a Committee using a Simple Majority" Econometrica. 16.

[10] Chichilnisky,G. "Manifolds of Preferences and Equilibria" (1976) Ph.D. Dissertation. Department of Economics, University of California, Berkeley.

[11] Chichilnisky, G. (1986) "Topological Complexity of Manifolds of Preferences" Chapter 8, Essays in Honor of Gerard Debreu (W. Hildenbrand and A. Mas-Colell eds.) North-Holland, 131-142.

[12] Chichilnisky, G.(1980) "Social Choice and the Topology of Spaces of Preferences" Advances in Mathematics, 37, No.2, 165-176.

[13] Chichilnisky, G. (1982) "Social Aggregation Rules and Continuity" Quarterly Journal of Economics, May, 337-352.

[14] Chichilnisky, G. (1993) "On Strategic Control" Quarterly Journal of Economics, February, 285-290.

[15] Chichilnisky, G. (1981) "Intersecting Families of Sets" in Essex Working Papers, University of Essex, U.K.

[16] Chichilnisky, G. (1992) "Limited Arbitrage is Necessary and Sufficient for the Existence of a Competitive Equilibrium", Working Paper No. 650, Department of Economics, Columbia University.

[17] Chichilnisky, G. (1992) "Topology and Economics: the Contribution of Stephen Smale", in From Topology to Computation, Proceedings of a Conference in Honor of S. Smale, (M. Hirsch, J. Marsden, and M Shub eds.) Springer Verlag, 1993, p. 147-161.

[18] Chichilnisky, G. (1992) "Markets, Arbitrage and Social Choice,' paper presented at the conference "Columbia Celebrates Arrow's Contributions" Columbia University, New York, October 27, 1991, Working Paper No. 586, Columbia University, December 1992, and CORE Discussion Paper No. 9342, CORE, Universite Catolique de Louvain, Louvain la Neuve, Belgium, 1993.

[19] Chichilnisky, G. (1993) "Intersecting Families of Sets and the Topology of Cones in Economics" Bulletin of the American Mathematical Society, Vol. 29, No. 2, October 1993, 189-207.

[20] Chichilnisky, G. (1995a) "A Unified Perspective on Resource Allocation: Limited Arbitrage is Necessary and Sufficient for the Existence of a Competitive Equilibrium, the Core and Social Choice," CORE Discussion Paper No. 9527, Universite Catolique de Louvain, Louvain-la-Neuve, Belgium, April 1995.

[21] Chichilnisky (1996) "Limited Arbitrage is Necessary and Sufficient for the Nonemptiness of the Core" Economic Letters, (in press), first published as "Limited Arbitrage is Necessary and Sufficient for the Existence of an Equilibrium and the Core and it Limits Voting Cycles", Economic Letters, in an issue which was reprinted without the paper December, Vol. 46, p. 321-331. 
[22] Chichilnisky, G. (1994) "Social Diversity and Gains from Trade: A Unified Perspective on Resource Allocation" American Economic Review, Papers and Proceedings, May 1994, Vol. 84, No. 4, p. 427-434.

[23] Chichilnisky, G. (1995) "A Unified Perspective on Resource Allocation: Limited Arbitrage is Necessary and Sufficient for the Existence of a Competitive Equilibrium. the Core and Social Choice" Invited presentation at the International Economic Association, May 1994, Hernstein Schloss, Vienna, Austria, forthcoming in Social Choice Reexamined, (eds. K. Arrow, A. Sen and T. Suzumura) McMillan.

[24] Chichilnisky, G. (1995) "Limited Arbitrage is Necessary and Sufficient for the Existence of a Competitive Equilibrium With or Without Short Sales", Economic Theory, Vol 5, No. 1, p. 79-108.

[25] Chichilnisky,G. (1996) "Limited Arbitrage is Necessary and Sufficient for the Existence of a Competitive Equilibrium With or Without Short Sales II" Working Paper, Columbia University, June 1996, Journal of Mathematical Economics, forthcoming.

[26] Chichilnisky, G. (1996) "A Topological Invariant for Competitive Markets" Working Paper, Columbia University, May 1996, Journal of Mathematical Economics, forthcoming.

[27] Chichilnisky, G. "Limited Arbitrage and Uniqueness of Equilibrium" Working Paper, Columbia University, July 1996.

[28] Chichilnisky, G. and G.M. Heal (1984) "Existence of a Competitive Equilibrium in $L_{p}$ and Sobolev spaces" IMA Preprint Series No. 79, Institute for Mathematics and its Applications, University of Minnesota, Minneapolis, MN, June 1984.

[29] Chichilnisky, G. and G. Heal (1993) "Existence. of a Competitive Equilibrium in Sobolev Spaces without Bounds on Short Sales" Journal of Economic Theory, vol $59,364-84$.

[30] Chichilnisky, G. and Heal G.M. (1992) "Equilibrium and the Core with Finitely or Infinitely Many Markets: A Unified Approach," forthcoming, Economic Theory.

[31] Chichilnisky, G. and G. M. Heal (1992) "Arbitrage and Equilibrium with Infinitely Many Securities and Commodities" First Boston Paper Series 92-29, July 1992, Columbia Business School, Columbia University.

[32] Chichilnisky, G. and G. Heal (1983) "Necessary and Sufficient Conditions for a Resolution of the Social Choice Paradox" Journal of Economic Theory, Vol 31, No. 1, 68-87.

[33] Condorcet, Marquis de (1785) Essai sur l'Application de l'Analyse a la Probabilite des Decisions Rendues a la Pluralite des Voix, Paris.

[34] Debreu, G. (1959) The Theory of Value, Cowles Foundation Monograph, John Wiley.

[35] Debreu, G. (1954) "Competitive Equilibrium and Pareto Optimum", Proceedings of the National Academy of Sciences 40, 588-92.

[36] Debreu, G. (1971) "Smooth Preferences" Econometrica 40, 603-615.

[37] Debreu, G. (1962) "New Concepts and Techniques for Equilibrium Analysis" International Economic Review, 3, 257-273. 
[38] Grandmont, J. M. (1982) 'Temporary Equilibrium' (Arrow K. and Intriligator M. eds.) Handbook of Mathematical Economics (New York: North Holland).

[39] Green. J. (1973) "Temporary Equilibrium in a Sequential Trading Model with Spot and Futures Transactions," Econometrica, Vol. 41, No. 6, pp. 1103-23.

[10] Hammond, P. (1983) "Overlapping Expectations and Hart's Conditions for Equilibrium in a Securities Market", Journal of Economic Theory, Vol. 31, pp. 170-75.

[41] Negishi, T. (1960) "Welfare Economics and the Existence of an Equilibrium for a Competitive Economy," Metroeconomica, Vol.

[42] Hart, O. (1974) "Existence of Equilibrium in a Securities Model", Journal of Economic Theory, Vol. 9, pp. 293-311.

[43] Heal, G. M. (1983) "Contractibility and Public Decision Making" Chapter 7, Social Choice and Welfare (eds. P. Pattanaik and M. Salles), North-Holland.

[44] Helly, E. (1933) "Uber Mengen Konvexen mit Gemeinschlaftlichen Punkten" $J$. Deutch Math. Verein, 32, 175-186.

[45] Kant, (1788) Critike der practischen Vernunft, English translation by L. W. Beck Critique of Practical Reason, Liberal Arts Press, New York, 1956.

[46] Kelley, J. (1960) General Topology, Van Nostrand, New York.

[47] McKenzie, L. (1959) "On the existence of a general equilibrium for competitive markets" Econometrica, 27, 54-71.

[48] McKenzie, L. (1987, 1989) "General Equilibrium", Chapter 1, General Equilibrium, The New Palgrave (eds. J. Eatwell, M. Milgate, P. Newman) Norton, New York.

[49] McKenzie, L.(1961) "On the Existence of General Equilibrium: Some Corrections" Econometrica, 29, 247-248.

[50] Monteiro, P., Page F. and Wooders M. (1996) "Arbitrage and Gains from Trade: A counterexample", mimeo, Journal of Mathematical Economics (forthcoming)

[51] Nielsen, L. (1989) "Asset Market Equilibrium with Short Selling" Review of Economic Studies, Vol. 56., No. 187, pp. 467-473.

[52] Nitzan,S. (1989) "More on the Preservation of Preference Proximity and Anonymous Social Choice" Quarterly Journal of Economics, 187-190.

[53] Page, F. H. Jr. (1987) "Notes and Comments to the Editor: On Equilibrium in Hart's Securities Exchange Model" Journal of Economic Theory, Vol. 41, No. 2, 392-404.

[54] Pattanaik, P. and A. Sen (1969) "Necessary and Sufficient Conditions for Rational Choice under Majority Decisions" Journal of Economic Theory, Vol 1, No. 2, August.

[55] Spanier, E. (1966) Algebraic Topology, McGraw Hill.

[56] Werner, J. (1987) "Arbitrage and the Existence of Competitive Equilibrium", Econometrica 55, No. 6, 1403-1418. 


\title{
1995-1996 Discussion Paper Series
}

\author{
Department of Economics \\ Columbia University \\ 1022 International Affairs Bldg. \\ 420 West 118th Street \\ New York, N.Y., 10027
}

The following papers are published in the 1995-96 Columbia University Discussion Paper series which runs from early November to October 31 of the following year (Academic Year).

Domestic orders for discussion papers are available for purchase at the cost of $\$ 8.00$ (U.S.) Per paper and $\$ 140.00$ (US) for the series.

Eoreign orders cost $\$ 10.00$ (US) per paper and $\$ 185.00$ for the series.

To order discussion papers, please write to the Discussion Paper Coordinator at the above address along with a check for the appropriate amount, made payable to Department of Economics, Columbia University. Please be sure to include the series number of the requested paper when you place an order. 


\section{5-96 Discussion Paper Series}

9596-01 Protectionist Response to Import Competition in Declining Industries Reconsidered

9596-02 New Estimates on Climate Demand: Evidence from Location Choice

9596-03 Enforcement by Hearing

9596-04 Preferential Trading Areas and Multilateralism: Strangers, Friends or Foes?

9596-05 Simplification, Progression and a Level Playing Field

9596-06 The Burden of Proof in Civil Litigation

9596-07 Market Structure and the Timing of Technology Adoption

9596-08 The Emergence of the World Economy

9596-09 The Global Age: From a Skeptical South to a Fearful North

9596-10 A Conformity Test for Cointegration

9596-11 Identification and Kullback Information in the GLSEM

9596-12 Informational Leverage and the Endogenous Timing of Product Introductions

9596-13 Changes in Wage Inequality

9596-14 The Design of Monte Carlo Experiments for VAR Models

9596-15 A Toplogical Invariant for Competitive Markets

9596-16 Topology and Invertible Maps by: J. Choi

by: M Cragg M Kahn

by: C. Sanchirico

by: J. Bhagwati

A. Panagariya

by: W Vickrey

by: C. Sanchirico

by: J. Choi M Thum

by: R. Findlay

by: J. Bhagwati

by: P. Dhrymes

by: P. Dhrymes

by: J. Choi

by: J. Mincer

by: P. Dhrymes

by: G. Chichilnisky

by: G. Chichilnisky

by: G. Chichilnisky 


\section{5-96 Discussion Paper Series}

9596-18 Measuring Neighborhood Investments: Urban Quality of Life

Expenditures by Race

by: D. Dipasquale

M Kahn

9596-19 The Silver Lining of Industrial Decline: Rust Belt Manufacturing's

Impact on Particulates

by: M Kahn

9596-20 Education's Role in Explaining Diabetic Health Investment Differentials

by: M Kahn

9596-21 Limited Arbitrage and Uniqueness of Market Equilibrium in Strictly Regular Economies

by: G. Chichilnisky

9596-22 A Probabilistic Model of Learning in Games

by: C. Sanchirico

9596-23 Minimal inclusive Sets in Special Classes of Games

by: C. Sanichirico

9596-24 'Globalization' and Vertical Structure

by: J. McLaren

9596-25 Corruption, Black Markets and the Fiscal Problem in LDC's: Some Recent Findings

by: J. McLaren

9596-26 Black Markets and Optimal Evadable Taxation

by: J. McLaren

9596-27 What Drives Deforestation in the Brazilian Amazon? Evidence from Satellite and Socioeconomic Data

by: A. Pfaff

9596-28 A Unified Perspective on Resource Allocation: Limited Arbitage is Necessary and Sufficient for the Existence of a Competitive Equilibrium, the Core and Social Choice

9596-29 Smooth Infinite Economies

by: G. Chichilnisky Y. Zhou

9596-30 Market Arbitage, Social Choice and the Core

by: G. Chichilnisky

9596-31 The Structural Barrier to Transition: A Note on Input-Output Tables of by: R. Ericson Centrally Planned Economies

9596-32 When and How to Vote: Resolving Non-contractible Uncertainty Efficiently

by: B. O'Flaherty

9596-33 The Form of U.S. In-Kind Assistance

by: B. O'Flaherty

9596-34 Mixing Government with Voluntaryism

by: $\mathrm{K}$. Lancaster 